\title{
Asymptotic Symmetries in the Gauge Fixing Approach and the BMS Group
}

\author{
Romain Ruzziconi* \\ Université Libre de Bruxelles and International Solvay Institutes \\ CP 231, B-1050 Brussels, Belgium \\ E-mail: rruzzico@ulb.ac.be
}

\begin{abstract}
These notes are an introduction to asymptotic symmetries in gauge theories, with a focus on general relativity in four dimensions. We explain how to impose consistent sets of boundary conditions in the gauge fixing approach and how to derive the asymptotic symmetry parameters. The different procedures to obtain the associated charges are presented. As an illustration of these general concepts, the examples of four-dimensional general relativity in asymptotically (locally) (A) $\mathrm{dS}_{4}$ and asymptotically flat spacetimes are covered. This enables us to discuss the different extensions of the Bondi-Metzner-Sachs-van der Burg (BMS) group and their relevance for holography, soft gravitons theorems, memory effects, and black hole information paradox. These notes are based on lectures given at the XV Modave Summer School in Mathematical Physics.
\end{abstract}

XV Modave Summer School in Mathematical Physics - Modave2019

8-14 September 2019

Modave, Belgium

${ }^{*}$ Speaker. 


\section{Introduction}

Gauge theories are of major importance in physics since they are involved in the fundamental description of nature. The Standard Model of particle physics and the theory of general relativity are two examples of gauge theories offering never-equaled observational predictions for our universe. Furthermore, gauge theories provide a mathematical framework that enables us to understand the deepest foundations of our physical theories.

The study of asymptotic symmetries in gauge theories is an old subject that has recently known renewed interest. A first direction is motivated by the AdS/CFT correspondence where the asymptotic symmetries of the gravity theory in the bulk spacetime correspond to the global symmetries of the dual quantum field theory through the holographic dictionary [3,126,165, 167, 177]. A strong control on asymptotic symmetries allows us to investigate new interesting holographic dualities. A second direction is driven by the recently-established connections between asymptotic symmetries, soft theorems and memory effects [162]. These connections furnish crucial information about the infrared structure of quantized gauge theories. In gravity, they may be relevant to solve the longstanding problem of black hole information paradox [101, 102, 105-107].

Several approaches exist regarding asymptotic symmetries in gauge theories and the construction of associated charges. The aim of these notes is to provide a self-consistent introduction on how to impose boundary conditions in a generic gauge theory, derive the asymptotic symmetry algebra, and compute the associated surface charges. We discuss these points in the gauge fixing approach. Indeed, despite this approach being widely used in the literature, there are few references discussing the complete procedure for a general gauge theory. To illustrate the abstract definitions and relevant results, we discuss in detail the examples of general relativity in asymptotically (locally) (A) $\mathrm{dS}_{4}$ and asymptotically flat spacetimes. These examples are interesting because they involve all the subtleties of the procedure. The notes aim to be pedagogical and are based on lectures given at the XV Modave Summer School in Mathematical Physics.

In section 2, we briefly mention the different main frameworks to study asymptotic symmetries in gauge theories. Thereafter, in section 3, we focus on the gauge fixing approach. We explain the conditions under which a given gauge fixing is suitable to study asymptotic symmetries. Then, we discuss how to impose consistent boundary conditions, the associated solution space, and how to derive the asymptotic symmetry algebra. In section 4, after some digressions through the Noether procedure to construct charges associated with global symmetries, we explain what the analogue of this construction for gauge symmetries is. In particular, the Barnich-Brandt prescription is discussed and related to the covariant phase space methods in the context of diffeomorphism-invariant theories. In section 5, we review some recent applications of asymptotic symmetries in the context of holography and the infrared sector of gauge theories. Finally, these notes are accompanied by two appendices. Appendix A is a quick summary of the matching between Bondi and Fefferman-Graham gauges in general relativity. Appendix B contains some important definitions and conventions about the jet bundles and homotopy operators widely used in the text.

Many reviews related to asymptotic symmetries complementary to these notes exist in the literature: see, for example, [19,26, 63,134,137, 145, 153,162]. 


\section{Definitions of asymptotics}

Several frameworks exist to impose boundary conditions in gauge theories. Some of them are mentioned next.

\subsection{Geometric approach}

The geometric approach of boundary conditions was initiated by Penrose, who introduced the techniques of conformal compactification to study general relativity in asymptotically flat spacetimes at null infinity $[144,146]$. According to this perspective, the boundary conditions are defined by requiring that certain data on a fixed boundary be preserved. The asymptotic symmetry group $G$ is then defined as the quotient:

$$
G=\frac{\text { Gauge transformation preserving the boundary conditions }}{\text { Trivial gauge transformations }},
$$

where the trivial gauge transformations are the gauge transformations that reduce to the identity on the boundary. In other words, the asymptotic symmetry group is isomorphic to the group of gauge transformations induced on the boundary which preserve the given data. This is the weak definition of the asymptotic symmetry group. A stronger definition of the asymptotic symmetry group is given by the quotient (2.1), where the trivial gauge transformations are now the gauge transformations that have associated vanishing charges.

The geometric approach was essentially used in gravity theory and led to much progress in the study of symmetries and symplectic structures for asymptotically flat spacetimes at null infinity $[6,7,83,103,114]$ and spatial infinity $[4,5]$. It was also considered to study asymptotically (A)dS spacetimes [8-11]. Moreover, this framework was recently applied to study boundary conditions and associated phase spaces on null hypersurfaces [56].

The advantage of this approach is that it is manifestly gauge invariant, since we do not refer to any particular coordinate system to impose the boundary conditions. Furthermore, the geometric interpretation of the symmetries is transparent. The weak point is that the definition of boundary conditions is rigid. It is a non-trivial task to modify a given set of boundary conditions in this framework to highlight new asymptotic symmetries. It is often a posteriori that boundary conditions are defined in this framework, after having obtained the results in coordinates.

\subsection{Gauge fixing approach}

A gauge theory has redundant degrees of freedom. The gauge fixing approach consists in using the gauge freedom of the theory to impose some constraints on the fields. This enables one to quotient the field space to eliminate some of the unphysical or pure gauge redundancies in the theory. For a given gauge theory, an appropriate gauge fixing (where appropriate will be defined below) still allows some redundancy. For example, in electrodynamics, the gauge field $A_{\mu}$ transforms as $A_{\mu} \rightarrow A_{\mu}+\partial_{\mu} \alpha$ ( $\alpha$ is a function of the spacetime coordinates) under a gauge transformation. The Lorenz gauge is defined by setting $\partial_{\mu} A^{\mu}=0$. This gauge can always be reached using the gauge redundancy, since $\partial_{\mu} \partial^{\mu} \alpha=-\partial_{v} A^{v}$ always admits a solution for $\alpha$, regardless of the exact form of $A_{\mu}$. However, residual gauge transformations remain that preserve the Lorenz gauge. These are given by $A_{\mu} \rightarrow A_{\mu}+\partial_{\mu} \beta$, where $\beta$ is a function of the spacetime coordinates satisfying $\partial_{\mu} \partial^{\mu} \beta=0$ 
(see, e.g., [119]). The same phenomenon occurs in general relativity where spacetime diffeomorphisms can be performed to reach a particular gauge defined by some conditions imposed on the metric $g_{\mu \nu}$. Some explicit examples are discussed below.

Then, the boundary conditions are imposed on the fields of the theory written in the chosen gauge. The weak version of the definition of the asymptotic symmetry group is given by

$$
G_{\text {weak }}=\left[\begin{array}{l}
\text { Residual gauge diffeomorphisms } \\
\text { preserving the boundary conditions. }
\end{array}\right]
$$

Intuitively, the gauge fixing procedure eliminates part of the pure gauge degrees of freedom, namely, the trivial gauge transformations defined under (2.1). Therefore, fixing the gauge is similar to taking the quotient as in equation (2.1), and the two definitions of asymptotic symmetry groups coincide in most of the practical situations. As in the geometric approach, a stronger version of the asymptotic symmetry group exists and is given by

$$
G_{\text {strong }}=\left[\begin{array}{l}
\text { Residual gauge diffeomorphisms preserving the boundary } \\
\text { conditions with associated non-vanishing charges. }
\end{array}\right]
$$

Notice that $G_{\text {strong }} \subseteq G_{\text {weak }}{ }^{1}$.

The advantage of the gauge fixing approach is that it is highly flexible to impose boundary conditions, since we are working with explicit expressions in coordinates. For example, the BMS group in four dimensions was first discovered in this framework [43, 154, 155]. Furthermore, a gauge fixing is a local consideration (i.e. it holds in a coordinate patch of the spacetime). Therefore, the global considerations related to the topology are not directly relevant in this analysis, thereby allowing further flexibility. For example, as we will discuss in subsection 3.4, this allowed to consider singular extensions of the BMS group: the Virasoro $\times$ Virasoro superrotations $[37,38]$. These new asymptotic symmetries are well-defined locally; however, they have poles on the celestial sphere. In the geometric approach, one would have to modify the topology of the spacetime boundary to allow these superrotations by considering some punctured celestial sphere $[21,164]$. The weakness of this approach is that it is not manifestly gauge invariant. Hence, even if the gauge fixing approach is often preferred to unveil new boundary conditions and symmetries, the geometric approach is complementary and necessary to make the gauge invariance of the results manifest. In section 3, we study the gauge fixing approach and provide some examples related to gravity in asymptotically flat and asymptotically (A)dS spacetimes.

\subsection{Hamiltonian approach}

Some alternative approaches exist that are also powerful in practice. For example, in the Hamiltonian formalism, asymptotically flat $[151]$ and $\operatorname{AdS}[47,110]$ spacetimes have been studied at spatial infinity. Furthermore, the global BMS group was recently identified at spatial infinity using twisted parity conditions [111-113]. In this framework, the computations are done in a coordinate system making the split between space and time explicit, without performing any gauge

\footnotetext{
${ }^{1}$ One of the most striking examples of the difference between the weak and the strong definitions of the asymptotic symmetry group is given by considering Neumann boundary conditions in asymptotically $\mathrm{AdS}_{d+1}$ spacetimes. Indeed, in this situation, we have $G_{\text {weak }}=\operatorname{Diff}\left(\mathbb{R} \times S^{d-1}\right)$, and $G_{\text {strong }}$ is trivial [69].
} 
fixing. Then, the asymptotic symmetry group is defined as the quotient between the gauge transformations preserving the boundary conditions and the trivial gauge transformations, where trivial means that the associated charges are identically vanishing on the phase space. This definition of the asymptotic symmetry group corresponds to the strong definition in the two first approaches.

\section{Asymptotic symmetries in the gauge fixing approach}

We now focus on the aforementioned gauge fixing approach of asymptotic symmetries in gauge theories. We illustrate the different definitions and concepts using examples, with a specific focus on asymptotically flat and asymptotically (A)dS spacetimes in four-dimensional general relativity.

\subsection{Gauge fixing procedure}

Definition [Gauge symmetry] Let us start with a Lagrangian theory in a $n$-dimensional spacetime $M$

$$
S[\Phi]=\int_{M} \mathbf{L}\left[\Phi, \partial_{\mu} \Phi, \partial_{\mu} \partial_{v} \Phi, \ldots\right]
$$

where $\mathbf{L}=L \mathrm{~d}^{n} x$ is the Lagrangian and $\Phi=\left(\phi^{i}\right)$ are the fields of the theory. A gauge transformation is a transformation acting on the fields, and which depends on parameters $F=\left(f^{\alpha}\right)$ that are taken to be arbitrary functions of the spacetime coordinates. We write

$$
\begin{aligned}
\delta_{F} \Phi & =R[F] \\
& =R_{\alpha} f^{\alpha}+R_{\alpha}^{\mu} \partial_{\mu} f^{\alpha}+R_{\alpha}^{(\mu v)} \partial_{\mu} \partial_{v} f^{\alpha}+\ldots \\
& =\sum_{k \geq 0} R_{\alpha}^{\left(\mu_{1} \ldots \mu_{k}\right)} \partial_{\mu_{1}} \ldots \partial_{\mu_{k}} f^{\alpha}
\end{aligned}
$$

the infinitesimal gauge transformation of the fields. In this expression, $R_{\alpha}^{\left(\mu_{1} \ldots \mu_{k}\right)}$ are local functions, namely functions of the coordinates, the fields, and their derivatives. The gauge transformation is a symmetry of the theory if, under (3.2), the Lagrangian transforms as

$$
\delta_{F} \mathbf{L}=\mathrm{d} \mathbf{B}_{F},
$$

where $\mathbf{B}_{F}=B_{F}^{\mu}\left(\mathrm{d}^{n-1} x\right)_{\mu}$.

Examples We illustrate this definition by providing some examples. First, consider classical vacuum electrodynamics

$$
S[A]=\int_{M} \mathbf{F} \wedge \star \mathbf{F},
$$

where $\mathbf{F}=\mathrm{d} \mathbf{A}$ and $\mathbf{A}$ is a 1-form. It is straightforward to check that the gauge transformation $\delta_{\alpha} \mathbf{A}=\mathrm{d} \alpha$, where $\alpha$ is an arbitrary function of the coordinates, is a symmetry of the theory.

Now, consider the general relativity theory

$$
S[g]=\frac{1}{16 \pi G} \int_{M}(R-2 \Lambda) \sqrt{-g} \mathrm{~d}^{n} x,
$$

where $R$ and $\sqrt{-g}$ are the scalar curvature and the square root of minus the determinant associated with the metric $g_{\mu \nu}$ respectively, and $\Lambda$ is the cosmological constant. It can be checked that the 
gauge transformation $\delta_{\xi} g_{\mu v}=\mathscr{L}_{\xi} g_{\mu v}=\xi^{\rho} \partial_{\rho} g_{\mu \nu}+g_{\mu \rho} \partial_{\nu} \xi^{\rho}+g_{\rho v} \partial_{\mu} \xi^{\rho}$, where $\xi^{\mu}$ is a vector field generating a diffeomorphism, is a symmetry of the theory.

Notice that in these examples, the transformation of the fields (3.2) is of the form

$$
\delta_{F} \Phi=R_{\alpha} f^{\alpha}+R_{\alpha}^{\mu} \partial_{\mu} f^{\alpha}
$$

namely they involve at most first order derivatives of the parameters.

Definition [Gauge fixing] Starting from a Lagrangian theory (3.1) with gauge symmetry (3.2), the gauge fixing procedure involves imposing some algebraic or differential constraints on the fields in order to eliminate (part of) the redundancy in the description of the theory. We write

$$
G[\Phi]=0
$$

a generic gauge fixing condition. This gauge has to satisfy two conditions:

- It has to be reachable by a gauge transformation, which means that the number of independent conditions in (3.7) is inferior or equal to the number of independent parameters $F=\left(f^{\alpha}\right)$ generating the gauge transformation.

- It has to use all of the available freedom of the arbitrary functions parametrizing the gauge transformations to reach the gauge ${ }^{2}$, which means that the number of independent conditions in (3.7) is superior or equal to the number of independent parameters $F=\left(f^{\alpha}\right)$ generating the gauge transformations.

Considering these two requirements together tells us that the number of independent gauge fixing conditions in (3.7) has to be equal to the number of independent gauge parameters $F=\left(f^{\alpha}\right)$ involved in the fields transformation (3.2).

Examples In electrodynamics, several gauge fixings are commonly used. Let us mention the Lorenz gauge $\partial^{\mu} A_{\mu}=0$, the Coulomb gauge $\partial^{i} A_{i}=0$, the temporal gauge $A_{0}=0$, and the axial gauge $A_{3}=0$. As previously discussed, the Lorenz gauge can always be reached by performing a gauge transformation. We can check that the same statement holds for all the other gauge fixings. Notice that these gauge fixing conditions involve only one constraint, as there is only one free parameter $\alpha$ in the gauge transformation.

In gravity, many gauge fixings are also used in practice. For example, the De Donder (or harmonic) gauge requires that the coordinates $x^{\mu}$ be harmonic functions, namely, $\square x^{\mu}=\frac{1}{\sqrt{-g}} \partial_{v}\left(\sqrt{-g} \partial^{v} x^{\mu}\right)=$ 0 . Notice that the number of constraints, $n$, is equal to the number of independent gauge parameters $\xi^{\mu}$. This gauge condition is suitable for studying gravitational waves in perturbation theory (see, e.g., [41]).

Another important gauge fixing in configurations where $\Lambda \neq 0$ is the Fefferman-Graham gauge $[89,90,139,157,158]$. We write the coordinates as $x^{\mu}=\left(\rho, x^{a}\right)$, where $a=1, \ldots, n-1$ and $\rho$ is an

\footnotetext{
${ }^{2}$ If the available freedom is not used, we talk about partial gauge fixing. In this configuration, there are still some arbitrary functions of the coordinates in the parameters of the residual gauge transformations.
} 
expansion parameter ( $\rho=0$ is at the spacetime boundary, and $\rho>0$ is in the bulk). It is defined by the following conditions:

$$
g_{\rho \rho}=-\frac{(n-1)(n-2)}{2 \Lambda \rho^{2}}, \quad g_{\rho a}=0
$$

( $n$ conditions). The coordinate $\rho$ is spacelike for $\Lambda<0$ and timelike for $\Lambda>0$. The most general metric takes the form

$$
\mathrm{d} s^{2}=-\frac{(n-1)(n-2)}{2 \Lambda} \frac{\mathrm{d} \rho^{2}}{\rho^{2}}+g_{a b}\left(\rho, x^{c}\right) \mathrm{d} x^{a} \mathrm{~d} x^{b} .
$$

Finally, the Bondi gauge will be relevant for us in the following [43,154, 155]. This gauge fixing is valid for both $\Lambda=0$ and $\Lambda \neq 0$ configurations. Writing the coordinates as $\left(u, r, x^{A}\right)$, where $x^{A}=\left(\theta_{1}, \ldots, \theta_{n-2}\right)$ are the transverse angular coordinates on the $(n-2)$-celestial sphere, the Bondi gauge is defined by the following conditions:

$$
g_{r r}=0, \quad g_{r A}=0, \quad \partial_{r}\left(\frac{\operatorname{det} g_{A B}}{r^{2(n-2)}}\right)=0
$$

( $n$ conditions). These conditions tell us that, geometrically, $u$ labels null hypersurfaces in the spacetime, $x^{A}$ labels null geodesics inside a null hypersurface, and $r$ is the luminosity distance along the null geodesics. The most general metric takes the form

$$
\mathrm{d} s^{2}=e^{2 \beta} \frac{V}{r} \mathrm{~d} u^{2}-2 e^{2 \beta} \mathrm{d} u \mathrm{~d} r+g_{A B}\left(\mathrm{~d} x^{A}-U^{A} \mathrm{~d} u\right)\left(d x^{B}-U^{B} \mathrm{~d} u\right)
$$

where $\beta, U^{A}$ and $\frac{V}{r}$ are arbitrary functions of the coordinates, and the $(n-2)$-dimensional metric $g_{A B}$ satisfies the determinant condition in the third equation of (3.10). Let us mention that the Bondi gauge is closely related to the Newman-Unti gauge $[29,135]$ involving only algebraic conditions:

$$
g_{r r}=0, \quad g_{r A}=0, \quad g_{r u}=-1
$$

( $n$ conditions).

Definition [Residual gauge transformation] After having imposed a gauge fixing as in equation (3.7), there usually remain some residual gauge transformations, namely gauge transformations preserving the gauge fixing condition. Formally, the residual gauge transformations with generators $F$ have to satisfy $\delta_{F} G[\Phi]=0$. They are local functions parametrized as $F=F(a)$, where the parameters $a$ are arbitrary functions of $(n-1)$ coordinates.

Examples Consider the Lorenz gauge $\partial^{\mu} A_{\mu}=0$ in electrodynamics. As we discussed earlier, the residual gauge transformations for the Lorenz gauge are the gauge transformations $\delta_{\alpha} A_{\mu}=\partial_{\mu} \alpha$, where $\partial^{\mu} \partial_{\mu} \alpha=0$.

Similarly, consider the Fefferman-Graham gauge (3.8) in general relativity with $\Lambda \neq 0$. The residual gauge transformations generated by $\xi^{\mu}$ have to satisfy $\mathscr{L}_{\xi} g_{\rho \rho}=0$ and $\mathscr{L}_{\xi} g_{\rho a}=0$. The solutions to these equations are given by

$$
\xi^{\rho}=\sigma\left(x^{a}\right) \rho, \quad \xi^{a}=\xi_{0}^{a}\left(x^{b}\right)+\frac{(n-1)(n-2)}{2 \Lambda} \partial_{b} \sigma \int_{0}^{\rho} \frac{\mathrm{d} \rho^{\prime}}{\rho^{\prime}} g^{a b}\left(\rho^{\prime}, x^{c}\right) .
$$


These solutions are parametrized by $n$ arbitrary functions $\sigma$ and $\xi_{0}^{a}$ of $(n-1)$ coordinates $x^{a}$.

In the Bondi gauge (3.10), the residual gauge transformations generated by $\xi^{\mu}$ have to satisfy $\mathscr{L}_{\xi} g_{r r}=0, \mathscr{L}_{\xi} g_{r A}=0$ and $g^{A B} \mathscr{L}_{\xi} g_{A B}=4 \omega$, where $\omega$ is an arbitrary function of $\left(u, x^{A}\right)$. The solutions to these equations are given by

$$
\begin{aligned}
& \xi^{u}=f \\
& \xi^{A}=Y^{A}+I^{A}, \quad I^{A}=-\partial_{B} f \int_{r}^{\infty} \mathrm{d} r^{\prime}\left(e^{2 \beta} g^{A B}\right), \\
& \xi^{r}=-\frac{r}{n-2}\left(\mathscr{D}_{A} Y^{A}-2 \omega+\mathscr{D}_{A} I^{A}-\partial_{B} f U^{B}+\frac{1}{2} f g^{-1} \partial_{u} g\right),
\end{aligned}
$$

where $\partial_{r} f=0=\partial_{r} Y^{A}$, and $g=\operatorname{det}\left(g_{A B}\right)$ [30]. The covariant derivative $\mathscr{D}_{A}$ is associated with the $(n-2)$-dimensional metric $g_{A B}$. The residual gauge transformations are parametrized by the $n$ functions $\omega, f$ and $Y^{A}$ of $(n-1)$ coordinates $\left(u, x^{A}\right)$.

\subsection{Boundary conditions}

Definition [Boundary conditions] Once a gauge condition (3.7) has been fixed, we can impose boundary conditions for the theory by requiering some constraints on the fields in a neighbourhood of a given spacetime region. Most of those boundary conditions are fall-off conditions on the fields in the considered asymptotic region ${ }^{3}$, or conditions on the leading functions in the expansion. This choice of boundary conditions is motivated by the physical model that we want to consider. A set of boundary conditions is usually considered to be interesting if it provides non-trivial asymptotic symmetry group and solution space, exhibiting interesting properties for the associated charges (finite, generically non-vanishing, integrable and conserved; see below). If the boundary conditions are too strong, the asymptotic symmetry group will be trivial, with vanishing surface charges. Furthermore, the solution space will not contain any solution of interest. If they are too weak, the associated surface charges will be divergent. Consistent and interesting boundary conditions should therefore be located between these two extreme situations.

Examples Let us give some examples of boundary conditions in general relativity theory. Many examples of boundary conditions for other gauge theories can be found in the literature (see e.g. $[2,30,55,75,109,124,160])$.

Let us consider the Bondi gauge defined in equation (3.10) in dimension $n \geq 3$. There exist several definitions of asymptotic flatness at null infinity $(r \rightarrow \infty)$ in the literature. For all of them, we require the following preliminary boundary conditions on the functions of the metric (3.11) in the asymptotic region $r \rightarrow \infty$ :

$$
\beta=o(1), \quad \frac{V}{r}=o\left(r^{2}\right), \quad U^{A}=o(1), \quad g_{A B}=r^{2} q_{A B}+r C_{A B}+D_{A B}+\mathscr{O}\left(r^{-1}\right),
$$

where $q_{A B}, C_{A B}$ and $D_{A B}$ are $(n-2)$-dimensional symmetric tensors, which are functions of $\left(u, x^{A}\right)$. Notice in particular that $q_{A B}$ is kept free at this stage.

\footnotetext{
${ }^{3}$ Notice that the asymptotic region could be taken not only at (spacelike, null or timelike) infinity, but also in other spacetime regions, such as near a black hole horizon [77-79,81,99, 101,102,106, 107].
} 
A first definition of asymptotic flatness at null infinity (AF1) is a sub-case of (3.15). In addition to all these fall-off conditions, we require the transverse boundary metric $q_{A B}$ to have a fixed determinant, namely,

$$
\sqrt{q}=\sqrt{\bar{q}},
$$

where $\bar{q}$ is a fixed volume element (which may possibly depend on time) on the $(n-2)$-dimensional transverse space $[51,52,65,94]$.

A second definition of asymptotic flatness at null infinity (AF2) is another sub-case of the definition (3.15). All the conditions are the same, except that we require that the transverse boundary metric $q_{A B}$ be conformally related to the unit $(n-2)$-sphere metric, namely,

$$
q_{A B}=e^{2 \varphi} \stackrel{\leftrightarrow}{A B}_{A},
$$

where $\stackrel{\circ}{q B}_{A B}$ is the unit $(n-2)$-sphere metric [36]. Note that for $n=4$, this condition can always be reached by a coordinate transformation, since every metric on a two dimensional surface is conformally flat (but even in this case, as we will see below, this restricts the form of the symmetries).

A third definition of asymptotic flatness at null infinity (AF3), which is the historical one $[43,154,155]$, is a sub-case of the second definition (3.17). We require (3.15) and we demand that the transverse boundary metric $q_{A B}$ be the unit $(n-2)$-sphere metric, namely,

$$
q_{A B}=\stackrel{\circ}{q}_{A B} .
$$

Note that this definition of asymptotic flatness is the only one that has the property to be asymptotically Minkowskian, that is, for $r \rightarrow \infty$, the leading orders of the spacetime metric (3.11) tend to the Minkowski line element $\mathrm{d} s^{2}=-\mathrm{d} u^{2}-2 \mathrm{~d} u \mathrm{~d} r+r^{2} \stackrel{\leftrightarrow}{q}_{A B} \mathrm{~d} x^{A} \mathrm{~d} x^{B}$.

Let us now present several definitions of asymptotically (A)dS spacetimes in both the Fefferman Graham gauge (3.8) and Bondi gauge (3.10). A preliminary boundary condition, usually called the asymptotically locally (A)dS condition, requires the following conditions on the functions of the Fefferman-Graham metric (3.9):

$$
g_{a b}=\mathscr{O}\left(\rho^{-2}\right)
$$

or, equivalently, $g_{a b}=\rho^{-2} g_{a b}^{(0)}+o\left(\rho^{-2}\right)$. Notice that the $(n-1)$-dimensional boundary metric $g_{a b}^{(0)}$ is kept free in this preliminary set of boundary conditions, thus justifying the adjective "locally" [130]. In the Bondi gauge, as we will see below, these fall-off conditions are equivalent to demand that

$$
g_{A B}=\mathscr{O}\left(r^{2}\right)
$$

or, equivalently, $g_{A B}=r^{2} q_{A B}+o\left(r^{2}\right)$.

A first definition of asymptotically (A)dS spacetime (AAdS1) is a sub-case of the definition (3.19). In addition to these fall-off conditions, we demand the following constraints on the $(n-1)$ dimensional boundary metric $g_{a b}^{(0)}$ :

$$
g_{t t}^{(0)}=\frac{2 \Lambda}{(n-1)(n-2)}, \quad g_{t A}^{(0)}=0, \quad \operatorname{det}\left(g_{a b}^{(0)}\right)=\frac{2 \Lambda}{(n-1)(n-2)} \bar{q},
$$


where $\bar{q}$ is a fixed volume form for the transverse $(n-2)$-dimensional space (which may possibly depend on $t$ ) [66]. In the Bondi gauge, the boundary conditions (3.21) translate into

$$
\beta=o(1), \quad \frac{V}{r}=\frac{2 r^{2} \Lambda}{(n-1)(n-2)}+o\left(r^{2}\right), \quad U^{A}=o(1), \quad \sqrt{q}=\sqrt{\bar{q}} .
$$

Notice the similarity of these conditions to the definition (AF1) (equations (3.15) and (3.16)) of asymptotically flat spacetime.

A second definition of asymptotically AdS spacetime ${ }^{4}$ (AAdS2) is a sub-case of the definition (3.19). We require the same conditions as in the preliminary boundary condition (3.19), except that we demand that the $(n-1)$-dimensional boundary metric $g_{a b}^{(0)}$ be fixed [110]. These conditions are called Dirichlet boundary conditions. One usually chooses the cylinder metric as the boundary metric, namely,

$$
g_{a b}^{(0)} \mathrm{d} x^{a} \mathrm{~d} x^{b}=\frac{2 \Lambda}{(n-1)(n-2)} \mathrm{d} t^{2}+\stackrel{\circ}{q}_{A B} \mathrm{~d} x^{A} \mathrm{~d} x^{B},
$$

where $\stackrel{\circ}{A B}_{A B}$ are the components of the unit $(n-2)$-sphere metric (as in the Bondi gauge, the upper case indices $A, B, \ldots$ run from 3 to $n$, and $x^{a}=\left(t, x^{A}\right)$ ). In the Bondi gauge, the boundary conditions (3.23) translate into

$$
\beta=o(1), \quad \frac{V}{r}=\frac{2 r^{2} \Lambda}{(n-1)(n-2)}+o\left(r^{2}\right), \quad U^{A}=o(1), \quad q_{A B}=\stackrel{\circ}{q}_{A B} .
$$

Notice the similarity of these conditions to the definition (AF3) (equations (3.15) and (3.18)) of asymptotically flat spacetime.

As we see it, the Bondi gauge is well-adapted for each type of asymptotics (see figure 1), while the Fefferman-Graham gauge is only defined in asymptotically (A)dS spacetimes.

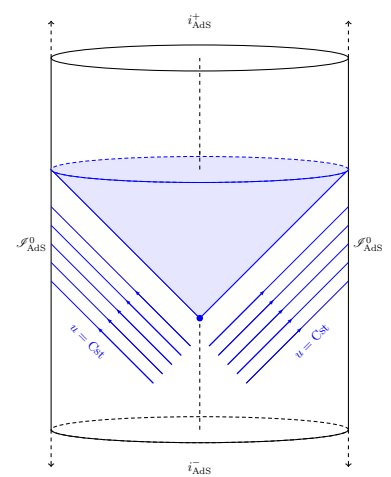

AdS case $(\Lambda<0)$.

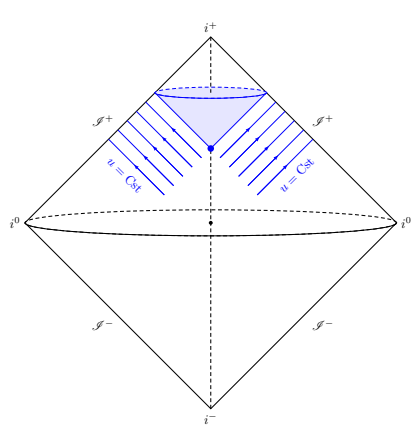

Flat case $(\Lambda=0)$.

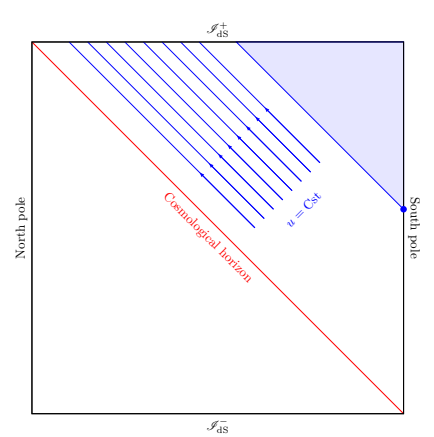

dS case $(\Lambda>0)$.

Figure 1: Bondi gauge for any $\Lambda$.

\footnotetext{
${ }^{4}$ This choice is less relevant for asymptotically dS spacetimes, since it strongly restricts the Cauchy problem and the bulk spacetime dynamics $[9,10]$.
} 


\subsection{Solution space}

Definition [Solution space] Given a gauge fixing (3.7) and boundary conditions, a solution of the theory is a field configuration $\tilde{\Phi}$ satisfying $G[\tilde{\Phi}]=0$, the boundary conditions, and the Euler Lagrange-equations

$$
\left.\frac{\delta \mathbf{L}}{\delta \Phi}\right|_{\tilde{\Phi}}=0
$$

where the Euler-Lagrange derivative is defined in equation (B.5). The set of all solutions of the theory is called the solution space. It is parametrized as $\tilde{\Phi}=\tilde{\Phi}(b)$, where the parameters $b$ are arbitrary functions of $(n-1)$ coordinates.

Examples We now provide some examples of solution spaces of four-dimensional general relativity in different gauge fixings. We first consider the Fefferman-Graham gauge in asymptotically (A) $\mathrm{dS}_{4}$ spacetimes with preliminary boundary conditions (3.19). Solving the Einstein equations

$$
G_{\mu v}+\Lambda g_{\mu v}=0
$$

we obtain the following analytic fall-offs:

$$
g_{a b}=\rho^{-2} g_{a b}^{(0)}+\rho^{-1} g_{a b}^{(1)}+g_{a b}^{(2)}+\rho g_{a b}^{(3)}+\mathscr{O}\left(\rho^{2}\right),
$$

where $g_{a b}^{(i)}$ are functions of $x^{a}[89,90,139,157,158]$. The only free data in this expansion are $g_{a b}^{(0)}$ and $g_{a b}^{(3)}$. All the other coefficients are determined in terms of these free data. Following the holographic dictionary, we call $g_{a b}^{(0)}$ the boundary metric and we define

$$
T_{a b}=\frac{\sqrt{3|\Lambda|}}{16 \pi G} g_{a b}^{(3)}
$$

as the stress energy tensor. From the Einstein equations, we have

$$
g_{a b}^{(0)} T^{a b}=0, \quad D_{a}^{(0)} T^{a b}=0
$$

where $D_{a}^{(0)}$ is the covariant derivative with respect to $g_{a b}^{(0)}$. In summary, the solution space of general relativity in the Fefferman-Graham gauge with the preliminary boundary condition (3.19) is parametrized by the set of functions

$$
\left\{g_{a b}^{(0)}, T_{a b}\right\}_{\Lambda \neq 0},
$$

where $T_{a b}$ satisfies (3.29) (11 functions).

Now, for the restricted set of boundary conditions (3.21), that is, (AAdS1), the solution space reduces to

$$
\left\{g_{A B}^{(0)}, T_{a b}\right\}_{\Lambda \neq 0},
$$

where $g_{A B}^{(0)}$ has a fixed determinant and $T_{a b}$ satisfies (3.29) (7 functions). Finally, for Dirichlet boundary conditions (3.23) (AAdS2), the solution space reduces to

$$
\left\{T_{a b}\right\}_{\Lambda \neq 0},
$$


where $T_{a b}$ satisfies (3.29) (5 functions).

Let us now consider the Bondi gauge in asymptotically $(\mathrm{A}) \mathrm{dS}_{4}$ spacetimes with preliminary boundary condition (3.20). From the Fefferman-Graham theorem and the gauge matching between Bondi and Fefferman-Graham that is described in appendix A (see also [66, 149]), we know that the functions appearing in the metric admit an analytic expansion in powers of $r$. In particular, we can write

$$
g_{A B}=r^{2} q_{A B}+r C_{A B}+D_{A B}+\frac{1}{r} E_{A B}+\frac{1}{r^{2}} F_{A B}+\mathscr{O}\left(r^{-3}\right),
$$

where $q_{A B}, C_{A B}, D_{A B}, E_{A B}, F_{A B}, \ldots$ are functions of $\left(u, x^{A}\right)$. The determinant condition defining the Bondi gauge and appearing in the third equation of (3.10) implies $g^{A B} \partial_{r} g_{A B}=4 / r$, which imposes successively that $\operatorname{det}\left(g_{A B}\right)=r^{4} \operatorname{det}\left(q_{A B}\right), q^{A B} C_{A B}=0$ and

$$
\begin{aligned}
D_{A B} & =\frac{1}{4} q_{A B} C^{C D} C_{C D}+\mathscr{D}_{A B}\left(u, x^{C}\right), \\
E_{A B} & =\frac{1}{2} q_{A B} \mathscr{D}_{C D} C^{C D}+\mathscr{E}_{A B}\left(u, x^{C}\right), \\
F_{A B} & =\frac{1}{2} q_{A B}\left[C^{C D} \mathscr{E}_{C D}+\frac{1}{2} \mathscr{D}^{C D} \mathscr{D}_{C D}-\frac{1}{32}\left(C^{C D} C_{C D}\right)^{2}\right]+\mathscr{F}_{A B}\left(u, x^{C}\right),
\end{aligned}
$$

with $q^{A B} \mathscr{D}_{A B}=q^{A B} \mathscr{E}_{A B}=q^{A B} \mathscr{F}_{A B}=0$ (indices are lowered and raised with the metric $q_{A B}$ and its inverse). We now sketch the results obtained by solving the Einstein equations

$$
G_{\mu v}+\Lambda g_{\mu v}=0
$$

for $\Lambda \neq 0$ (we follow [66, 149]; see also [127] for the Newman-Penrose version). The component $(r r)$ gives the following radial constraints on the Bondi functions:

$$
\begin{aligned}
\beta\left(u, r, x^{A}\right)=\beta_{0}\left(u, x^{A}\right) & +\frac{1}{r^{2}}\left[-\frac{1}{32} C^{A B} C_{A B}\right]+\frac{1}{r^{3}}\left[-\frac{1}{12} C^{A B} \mathscr{D}_{A B}\right] \\
+ & \frac{1}{r^{4}}\left[-\frac{3}{32} C^{A B} \mathscr{E}_{A B}-\frac{1}{16} \mathscr{D}^{A B} \mathscr{D}_{A B}+\frac{1}{128}\left(C^{A B} C_{A B}\right)^{2}\right]+\mathscr{O}\left(r^{-5}\right) .
\end{aligned}
$$

where $\beta_{0}\left(u, x^{A}\right)$ is an arbitrary function. The component $(r A)$ yields

$$
\begin{aligned}
U^{A}= & U_{0}^{A}\left(u, x^{B}\right)+\stackrel{(1)}{U^{A}}\left(u, x^{B}\right) \frac{1}{r}+\stackrel{(2)}{U^{A}}\left(u, x^{B}\right) \frac{1}{r^{2}} \\
& +U^{A}\left(u, x^{B}\right) \frac{1}{r^{3}}+{ }^{A} U^{A}\left(u, x^{B}\right) \frac{\ln r}{r^{3}}+o\left(r^{-3}\right)
\end{aligned}
$$

with

$$
\begin{aligned}
& \stackrel{(1)}{U^{A}}\left(u, x^{B}\right)=2 e^{2 \beta_{0}} \partial^{A} \beta_{0}, \\
& U^{A}\left(u, x^{B}\right)=-e^{2 \beta_{0}}\left[C^{A B} \partial_{B} \beta_{0}+\frac{1}{2} D_{B} C^{A B}\right], \\
& \stackrel{(3)}{U^{A}}\left(u, x^{B}\right)=-\frac{2}{3} e^{2 \beta_{0}}\left[N^{A}-\frac{1}{2} C^{A B} D^{C} C_{B C}+\left(\partial_{B} \beta_{0}-\frac{1}{3} D_{B}\right) \mathscr{D}^{A B}-\frac{3}{16} C_{C D} C^{C D} \partial^{A} \beta_{0}\right], \\
& U^{A}\left(u, x^{B}\right)=-\frac{2}{3} e^{2 \beta_{0}} D_{B} \mathscr{D}^{A B} \text {. }
\end{aligned}
$$


In these expressions, $U_{0}^{A}\left(u, x^{B}\right)$ and $N^{A}\left(u, x^{B}\right)$ are arbitrary functions. We call $N^{A}$ the angular momentum aspect. Notice that, at this stage, logarithmic terms are appearing in the expansion (3.37). However, we will see below that these terms vanish for $\Lambda \neq 0$. The component $(r u)$ leads to

$$
\begin{aligned}
\frac{V}{r}= & \frac{\Lambda}{3} e^{2 \beta_{0}} r^{2}-r\left(l+D_{A} U_{0}^{A}\right) \\
& -e^{2 \beta_{0}}\left[\frac{1}{2}\left(R[q]+\frac{\Lambda}{8} C_{A B} C^{A B}\right)+2 D_{A} \partial^{A} \beta_{0}+4 \partial_{A} \beta_{0} \partial^{A} \beta_{0}\right]-\frac{2 M}{r}+o\left(r^{-1}\right)
\end{aligned}
$$

where $l=\partial_{u} \ln \sqrt{q}, R[q]$ is the scalar curvature associated with the metric $q_{A B}$ and $M\left(u, x^{A}\right)$ is an arbitrary function called the Bondi mass aspect. Afterwards, we solve the components $(A B)$ of the Einstein equations order by order, thereby providing us with the constraints imposed on each order of $g_{A B}$. The leading order $\mathscr{O}\left(r^{-1}\right)$ of that equation yields to

$$
\frac{\Lambda}{3} C_{A B}=e^{-2 \beta_{0}}\left[\left(\partial_{u}-l\right) q_{A B}+2 D_{(A} U_{B)}^{0}-D^{C} U_{C}^{0} q_{A B}\right] .
$$

Going to $\mathscr{O}\left(r^{-2}\right)$, we get

$$
\frac{\Lambda}{3} \mathscr{D}_{A B}=0
$$

which removes the logarithmic term in (3.37) for $\Lambda \neq 0$ (but not for $\Lambda=0$ ). The condition at the next order $\mathscr{O}\left(r^{-3}\right)$

$$
\partial_{u} \mathscr{D}_{A B}+U_{0}^{C} D_{C} \mathscr{D}_{A B}+2 \mathscr{D}_{C(A} D_{B} U_{0}^{C}=0
$$

is trivial for $\Lambda \neq 0$. Using an iterative argument as in [149], we now make the following observation. If we decompose $g_{A B}=r^{2} \sum_{n \geq 0} g_{A B}^{(n)} r^{-n}$, we see that the iterative solution of the components $(A B)$ of the Einstein equations organizes itself as $\Lambda g_{A B}^{(n)}=\partial_{u} g_{A B}^{(n-1)}+(\ldots)$ at order $\mathscr{O}\left(r^{-n}\right), n \in \mathbb{N}_{0}$. Accordingly, the form of $\mathscr{E}_{A B}$ should have been fixed by the equation found at $\mathscr{O}\left(r^{-3}\right)$; however, this is not the case, since both contributions of $\mathscr{E}_{A B}$ cancel between $G_{A B}$ and $\Lambda g_{A B}$. Moreover, the equation $\Lambda g_{A B}^{(4)}=\partial_{u} g_{A B}^{(3)}+(\ldots)$ at the next order turns out to be a constraint for $g_{A B}^{(4)} \sim \mathscr{F}_{A B}$, determined with other subleading data such as $C_{A B}$ or $\partial_{u} g_{A B}^{(3)} \sim \partial_{u} \mathscr{E}_{A B}$. It shows that $\mathscr{E}_{A B}$ is a set of two free data on the boundary, built up from two arbitrary functions of $\left(u, x^{A}\right)$. Morover, it indicates that no more data exist to be uncovered for $\Lambda \neq 0$. Finally, the components $(u u)$ and $(u A)$ of the Einstein equations give some evolution constraints with respect to the $u$ coordinate for the Bondi mass aspect $M$ and the angular momentum aspect $N^{A}$. We will not describe these equations explicitly here (see $[66,149])$.

In summary, the solution space for general relativity in the Bondi gauge with the preliminary boundary condition (3.33) and $\Lambda \neq 0$ is parametrized by the set of functions

$$
\left\{\beta_{0}, U_{0}^{A}, q_{A B}, \mathscr{E}_{A B}, M, N^{A}\right\}_{\Lambda \neq 0}
$$

(11 functions), where $M$ and $N^{A}$ have constrained evolutions with respect to the $u$ coordinate. Therefore, the characteristic initial value problem is well-defined when the following data are given: $\beta_{0}\left(u, x^{C}\right), U_{0}^{A}\left(u, x^{C}\right), \mathscr{E}_{A B}\left(u, x^{C}\right), q_{A B}\left(u, x^{C}\right), M\left(u_{0}, x^{C}\right)$ and $N^{A}\left(u_{0}, x^{C}\right)$, where $u_{0}$ is a fixed value of $u$. 
Notice that for the boundary conditions (3.22) (AAdS1), the solution space reduces to

$$
\left\{q_{A B}, \mathscr{E}_{A B}, M, N^{A}\right\}_{\Lambda \neq 0},
$$

where $M$ and $N^{A}$ have constrained evolutions with respect to the $u$ coordinate, and $q_{A B}$ has a fixed determinant [66] (7 functions). Finally, for the Dirichlet boundary conditions (3.24) (AAdS2), the solution space finally reduces to

$$
\left\{\mathscr{E}_{A B}, M, N^{A}\right\}_{\Lambda \neq 0},
$$

where $M$ and $N^{A}$ have constrained evolutions with respect to the $u$ coordinate ( 5 functions).

Let us finally discuss the Bondi gauge in asymptotically flat spacetimes $[36,43,65,66,93,154$, 155]. We first consider the preliminary boundary conditions (3.15). From the previous analysis of solution space with $\Lambda \neq 0$, we can readily obtain the solution space with $\Lambda=0$, that is, the solution of

$$
G_{\mu v}=0,
$$

by taking the flat limit $\Lambda \rightarrow 0$. The radial constraints (3.36), (3.38) and (3.39) are still valid by setting to zero $\beta_{0}, U_{0}^{A}$ (see equation (3.15)) and all the terms proportional to $\Lambda$. Furthermore, by the same procedure, the constraint equation (3.40) becomes

$$
\left(\partial_{u}-l\right) q_{A B}=0 .
$$

Therefore, the asymptotic shear $C_{A B}$ becomes unconstrained, and the metric $q_{A B}$ gets a time evolution constraint. Similarly, the equation (3.41) becomes trivial and $\mathscr{D}_{A B}$ is not constrained at this order. In particular, this allows for the existence of logarithmic terms in the Bondi expansion (see equation (3.37)). One has to impose the additional condition $D^{A} \mathscr{D}_{A B}=0$ to make these logarithmic terms disappear. Finally, one can see that for $\Lambda=0$, the subleading orders of the components $(A B)$ of the Einstein equations impose time evolution constraints on $\mathscr{D}_{A B}, \mathscr{E}_{A B}, \ldots$, but this infinite tower of functions is otherwise unconstrained and they become free parameters of the solution space. Finally, as for the case $\Lambda \neq 0$, the components $(u u)$ and $(u A)$ of the Einstein equations yield time evolution constraints for the Bondi mass aspect $M$ and the angular momentum aspect $N^{A}$.

In summary, the solution space for general relativity in the Bondi gauge with the preliminary boundary condition (3.15) is parametrized by the set of functions

$$
\left\{q_{A B}, C_{A B}, M, N^{A}, \mathscr{D}_{A B}, \mathscr{E}_{A B}, \mathscr{F}_{A B}, \ldots\right\}_{\Lambda=0},
$$

where $q_{A B}, M, N^{A}, \mathscr{D}_{A B}, \mathscr{E}_{A B}, \mathscr{F}_{A B}, \ldots$ have constrained time evolutions (infinite tower of independent functions). Therefore, the characteristic initial value problem is well-defined when the following data are given: $C_{A B}\left(u, c^{C}\right), q_{A B}\left(u_{0}, x^{C}\right), M\left(u_{0}, x^{C}\right), N^{A}\left(u_{0}, x^{C}\right), \mathscr{D}_{A B}\left(u_{0}, x^{C}\right), \mathscr{E}_{A B}\left(u_{0}, x^{C}\right)$, $\mathscr{F}_{A B}\left(u_{0}, x^{C}\right), \ldots$ where $u_{0}$ is a fixed value of $u$. Notice a subtle point here: by taking the flat limit of the solution space with $\Lambda \neq 0$, we assumed that $g_{A B}$ is analytic in $r$ and can be expanded as (3.33) (this condition was not restrictive for $\Lambda \neq 0$ ). This condition is slightly more restrictive than (3.15) where analyticity is assumed only up to order $r^{-1}$. Therefore, by this flat limit procedure, we only obtain a subsector of the most general solution space. Writing $g_{A B}\left(u, r, x^{C}\right)=r^{2} q_{A B}\left(u, x^{C}\right)+r C_{A B}\left(u, x^{C}\right)+D_{A B}\left(u, x^{C}\right)+\tilde{E}_{A B}\left(u, r, x^{C}\right)$, where $\tilde{E}_{A B}$ is a function of all the coordinates of order $\mathscr{O}\left(r^{-1}\right)$ in $r$, the most general solution space can be written as

$$
\left\{q_{A B}, C_{A B}, M, N^{A}, \mathscr{D}_{A B}, \tilde{\mathscr{E}}_{A B}\right\}_{\Lambda=0},
$$


where $\tilde{\mathscr{E}}_{A B}$ is the trace-free part of $\tilde{E}_{A B}$, and $q_{A B}, M, N^{A}, \mathscr{D}_{A B}, \tilde{\mathscr{E}}_{A B}$ obey time evolution constraints. Now, the characteristic initial value problem is well-defined when the following data are given: $C_{A B}\left(u, c^{C}\right), q_{A B}\left(u_{0}, x^{C}\right), M\left(u_{0}, x^{C}\right), N^{A}\left(u_{0}, x^{C}\right), \mathscr{D}_{A B}\left(u_{0}, x^{C}\right)$ and $\tilde{\mathscr{E}}_{A B}\left(u_{0}, r, x^{C}\right)$.

We complete this set of examples by mentioning the restricted solution spaces in the different definitions of asymptotic flatness introduced above. For boundary conditions (AF1) (equations (3.15) with (3.16)), we obtain

$$
\left\{q_{A B}, C_{A B}, M, N^{A}, \mathscr{D}_{A B}, \tilde{\mathscr{E}}_{A B}\right\}_{\Lambda=0},
$$

where $q_{A B}, M, N^{A}, \mathscr{D}_{A B}$ and $\tilde{\mathscr{E}}_{A B}$ obey time evolution constraints, and $\sqrt{q}$ is fixed. In particular, if we choose a branch where $\sqrt{q}$ is time-independent, from (3.47), we immediately see that $\partial_{u} q_{A B}=$ $0^{5}$. For boundary conditions (AF2) (equations (3.15) with (3.17)), the solution space reduces to

$$
\left\{\varphi, C_{A B}, M, N^{A}, \mathscr{D}_{A B}, \tilde{\mathscr{E}}_{A B}\right\}_{\Lambda=0}
$$

where $M, N^{A}, \mathscr{D}_{A B}$ and $\tilde{\mathscr{E}}_{A B}$ obey time evolution equations. Notice that the metric $q_{A B}$ of the form (3.17) automatically satisfies (3.47). This agrees with results of [36]. Finally, taking the boundary conditions (AF3) (equations (3.15) with (3.18)) yields the solution space

$$
\left\{C_{A B}, M, N^{A}, \mathscr{D}_{A B}, \tilde{\mathscr{E}}_{A B}\right\}_{\Lambda=0}
$$

where $M, N^{A}, \mathscr{D}_{A B}$ and $\tilde{\mathscr{E}}_{A B}$ obey time evolution equations. This agrees with the historical results of $[43,154,155]$.

\subsection{Asymptotic symmetry algebra}

Definition [Asymptotic symmetry] Given boundary conditions imposed in a chosen gauge, the asymptotic symmetries are defined as the residual gauge transformations preserving the boundary conditions ${ }^{6}$. In other words, the asymptotic symmetries considered on-shell are the gauge transformations $R[F]$ tangent to the solution space. In practice, the requirement to preserve the boundary conditions gives some constraints on the functions parametrizing the residual gauge transformations. In gravity, the generators of asymptotic symmetries are often called asymptotic Killing vectors.

Definition [Asymptotic symmetry algebra] Once the asymptotic symmetries are known, we have

$$
\begin{aligned}
{\left[R\left[F_{1}\right], R\left[F_{2}\right]\right] } & =\delta_{F_{1}} R\left[F_{2}\right]-\delta_{F_{2}} R\left[F_{1}\right] \\
& \approx R\left[\left[F_{1}, F_{2}\right]_{A}\right]
\end{aligned}
$$

where $\approx$ means that this equality holds on-shell, i.e. on the solution space. In this expression, the bracket $\left[F_{1}, F_{2}\right]_{A}$ of gauge symmetry generators is given by

$$
\left[F_{1}, F_{2}\right]_{A}=C\left(F_{1}, F_{2}\right)-\delta_{F_{1}} F_{2}+\delta_{F_{2}} F_{1},
$$

\footnotetext{
${ }^{5}$ The condition $\partial_{u} q_{A B}=0$ was assumed for technical reasons in $[51,52,65]$, but this was actually not restrictive.

${ }^{6}$ This is the weak definition of asymptotic symmetry, in the sense of (2.2).
} 
where $C\left(F_{1}, F_{2}\right)$ is a skew-symmetric bi-differential operator $[21,26]$

$$
C\left(F_{1}, F_{2}\right)=\sum_{k, l \geq 0} C_{[\alpha \beta]}^{\left(\mu_{1} \cdots \mu_{k}\right)\left(v_{1} \cdots v_{l}\right)} \partial_{\mu_{1}} \ldots \partial_{\mu_{k}} f_{1}^{\alpha} \partial_{v_{1}} \ldots \partial_{v_{l}} f_{2}^{\beta} .
$$

The presence of the terms $-\delta_{F_{1}} F_{2}+\delta_{F_{2}} F_{1}$ in (3.53) is due to the possible field-dependence of the asymptotic symmetry generators. We can verify that (3.54) satisfies the Jacobi identity, i.e. the asymptotic symmetry generators form a (solution space-dependent) Lie algebra for this bracket. It is called the asymptotic symmetry algebra. The statement (3.53) means that the infinitesimal action of the gauge symmetries on the fields forms a representation of the Lie algebra of asymptotic symmetry generators: $\left[\delta_{F_{1}}, \delta_{F_{2}}\right] \Phi=\delta_{\left[F_{1}, F_{2}\right]} \Phi$. Let us mention that a Lie algebroid structure is showing up at this stage $[21,21,72]$. The base manifold is given by the solution space, the field-dependent Lie algebra is the Lie algebra of asymptotic symmetry generators introduced above and the anchor is the map $F \rightarrow R[F]$.

Examples Let us start by considering asymptotically $A d S_{4}$ spacetimes in the Fefferman-Graham and Bondi gauge. The preliminary boundary condition (3.19) does not impose any constraint on the generators of the residual gauge diffeomorphisms of the Fefferman-Graham gauge given in (3.13). Similarly, the generators of the residual gauge diffeomorphisms in Bondi gauge given in (3.14) do not get further constraints with (3.20).

Now, let us consider the boundary conditions (AAdS1) (equation (3.19) together with (3.21)) in the Fefferman-Graham gauge. The asymptotic symmetries are generated by the vectors fields $\xi^{\mu}$ given in (3.13) preserving the boundary conditions, namely, satisfying $\mathscr{L}_{\xi} g_{t t}^{(0)}=0, \mathscr{L}_{\xi} g_{t A}^{(0)}=0$ and $g_{(0)}^{a b} \mathscr{L}_{\xi} g_{a b}^{(0)}=0$. This leads to the following constraints on the parameters:

$$
\left(\partial_{u}-\frac{1}{2} l\right) \xi_{0}^{t}=\frac{1}{2} D_{A}^{(0)} \xi_{0}^{A}, \quad \partial_{u} \xi_{0}^{A}=-\frac{\Lambda}{3} g_{(0)}^{A B} \partial_{B} \xi_{0}^{t}, \quad \sigma=\frac{1}{2}\left(D_{A}^{(0)} \xi_{0}^{A}+\xi_{0}^{t} l\right),
$$

where $l=\partial_{u} \ln \sqrt{\bar{q}}$. In this case, the Lie bracket (3.54) is given by

$$
\left[\xi_{1}, \xi_{2}\right]_{A}=\mathscr{L}_{\xi_{1}} \xi_{2}-\delta_{\xi_{1}} \xi_{2}+\delta_{\xi_{2}} \xi_{1}
$$

and is referred as the modified Lie bracket [36]. Therefore, the asymptotic symmetry algebra can be worked out and is given explicitly by $\left[\xi\left(\xi_{0,1}^{t}, \xi_{0,1}^{A}\right), \xi\left(\xi_{0,2}^{t}, \xi_{0,2}^{A}\right)\right]_{A}=\xi\left(\hat{\xi}_{0}^{t}, \hat{\xi}_{0}^{A}\right)$, where

$$
\begin{aligned}
& \hat{\xi}_{0}^{t}=\xi_{0,1}^{A} \partial_{A} \xi_{0,2}^{t}+\frac{1}{2} \xi_{0,1}^{t} D_{A}^{(0)} \xi_{0,2}^{A}-\delta_{\xi\left(\xi_{0,1}^{t}, \xi_{0,1}^{A}\right.} \xi_{0,2}^{t}-(1 \leftrightarrow 2), \\
& \hat{\xi}_{0}^{A}=\xi_{0,1}^{B} \partial_{B} \xi_{0,2}^{A}-\frac{\Lambda}{3} \xi_{0,1}^{t} g_{(0)}^{A B} \partial_{B} \xi_{0,2}^{t}-\delta_{\xi\left(\xi_{0,1}^{t}, \xi_{0,1}^{A}\right.} \xi_{0,2}^{A}-(1 \leftrightarrow 2) .
\end{aligned}
$$

In the Bondi gauge with corresponding boundary conditions (3.22), the constraints on the parameters are given by

$$
\left(\partial_{u}-\frac{1}{2} l\right) f=\frac{1}{2} D_{A} Y^{A}, \quad \partial_{u} Y^{A}=-\frac{\Lambda}{3} q^{A B} \partial_{B} f, \quad \omega=0
$$

and the asymptotic symmetry algebra is written as $\left[\xi\left(f_{1}, Y_{1}^{A}\right), \xi\left(f_{2}, Y_{2}^{A}\right)\right]_{A}=\xi\left(\hat{f}, \hat{Y}^{A}\right)$ where

$$
\begin{aligned}
\hat{f} & =Y_{1}^{A} \partial_{A} f_{2}+\frac{1}{2} f_{1} D_{A} Y_{2}^{A}-\delta_{\xi\left(f_{1}, Y_{1}^{A}\right)} f_{2}-(1 \leftrightarrow 2), \\
\hat{Y}^{A} & =Y_{1}^{B} \partial_{B} Y_{2}^{A}-\frac{\Lambda}{3} f_{1} q^{A B} \partial_{B} f_{2}-\delta_{\xi\left(f_{1}, Y_{1}^{A}\right)} Y_{2}^{A}-(1 \leftrightarrow 2) .
\end{aligned}
$$


This asymptotic symmetry algebra is infinite-dimensional (in particular, it contains the area-preserving diffeomorphisms as a subgroup) and field-dependent, and it is called the $\Lambda-\mathrm{BMS}_{4}$ algebra [66]. The parameters $f$ are called the supertranslation generators, while the parameters $Y^{A}$ are called the superrotation generators. These names will be justified below when studying the flat limit of this asymptotic symmetry algebra $\Lambda$-BMS 4 . The computation of the modified Lie bracket (3.57) in the Bondi gauge for these boundary conditions ${ }^{7}$ follows closely [36].

Let us consider the Fefferman-Graham gauge with Dirichlet boundary conditions (AAdS2), that is, (3.19) together with (3.23). Compared to the above situation, the equations (3.56) reduce to

$$
\partial_{u} \xi_{0}^{t}=\frac{1}{2} D_{A}^{(0)} \xi_{0}^{A}, \quad \partial_{u} Y^{A}=-\frac{\Lambda}{3} \stackrel{\circ}{q}_{(0)}^{A B} \partial_{B} \xi_{0}^{t}, \quad \sigma=\frac{1}{2} D_{A}^{(0)} \xi_{0}^{A},
$$

where $D_{A}^{(0)}$ is the covariant derivative associated with the fixed unit sphere metric $\stackrel{\circ}{A B}_{A B}$. Furthermore, there is an additional constraint: $\mathscr{L}_{\xi} g_{A B}^{(0)}=o\left(\rho^{-2}\right)$, which indicates that $\xi_{0}^{A}$ is a conformal Killing vector of $\stackrel{\circ}{q}_{A B}$, namely,

$$
D_{A}^{(0)} \xi_{B}^{0}+D_{B}^{(0)} \xi_{A}^{0}=D_{C}^{(0)} \xi_{0}^{C} \stackrel{\circ}{q}_{A B}
$$

The asymptotic symmetry algebra remains of the same form as (3.58). In the Bondi gauge, Dirichlet boundary conditions are given by (3.20) together with (3.24). The equations (3.59) become

$$
\partial_{u} f=\frac{1}{2} D_{A} Y^{A}, \quad \partial_{u} Y^{A}=-\frac{\Lambda}{3} \stackrel{q}{q}^{A B} \partial_{B} \xi_{0}^{t}, \quad \omega=0,
$$

where $D_{A}$ is the covariant derivative with respect to $\stackrel{\circ}{A}_{A B}$, while the additional constraint $\mathscr{L}_{\xi} g_{A B}=$ $o\left(r^{2}\right)$ gives

$$
D_{A} Y_{B}+D_{B} Y_{A}=D_{C} Y^{C} \stackrel{\circ}{q}_{A B} .
$$

This means that $Y^{A}$ is a conformal Killing vector of $\stackrel{\circ}{q}_{A B}$. The asymptotic symmetry algebra (3.60) remains of the same form. It can be shown that the asymptotic symmetry algebra corresponds to $S O(3,2)$ algebra for $\Lambda<0$ and $S O(1,4)$ algebra for $\Lambda>0$ [30] (see also Appendix A of [66]). Therefore, we see how the infinite-dimensional asymptotic symmetry algebra $\Lambda$ - $\mathrm{BMS}_{4}$ reduces to these finite-dimensional algebras, which are the symmetry algebras of global $\mathrm{AdS}_{4}$ and global $\mathrm{dS}_{4}$, respectively.

Let us now consider asymptotically flat spacetimes in the Bondi gauge. The asymptotic Killing vectors can be derived in a similar way to that in the previous examples. Another way in which to proceed is to take the flat limit of the previous results obtained in the Bondi gauge. We sketch the expressions obtained by following these two equivalent procedures. First, consider the preliminary boundary conditions (3.15). The asymptotic Killing vectors $\xi^{\mu}$ are the residual gauge diffeomorphisms (3.14) with the following constraints on the parameters:

$$
\left(\partial_{u}-\frac{1}{2} l\right) f=\frac{1}{2} D_{A} Y^{A}-\omega, \quad \partial_{u} Y^{A}=0,
$$

where $l=\partial_{u} \ln \sqrt{q}$. These equations can be readily solved and the solutions are given by

$$
f=q^{\frac{1}{4}}\left[T\left(x^{A}\right)+\frac{1}{2} \int_{0}^{u} \mathrm{~d} u^{\prime}\left[q^{-\frac{1}{4}}\left(D_{A} Y^{A}-2 \omega\right)\right]\right], \quad Y^{A}=Y^{A}\left(x^{B}\right),
$$

\footnotetext{
${ }^{7}$ This completes the results obtained in [66] where the asymptotic symmetry algebra was obtained by pullback methods.
} 
where $T$ are called supertranslation generators and $Y^{A}$ superrotation generators. Notice that there is no additional constraint on $Y^{A}$ at this stage. Computing the modified Lie bracket (3.57), we obtain $\left[\xi\left(f_{1}, Y_{1}^{A}, \omega_{1}\right), \xi\left(f_{2}, Y_{2}^{A}, \omega_{2}\right)\right]_{A}=\xi\left(\hat{f}, \hat{Y}^{A}, \hat{\omega}\right)$ where

$$
\begin{aligned}
\hat{f} & =Y_{1}^{A} \partial_{A} f_{2}+\frac{1}{2} f_{1} D_{A} Y_{2}^{A}-(1 \leftrightarrow 2), \\
\hat{Y}^{A} & =Y_{1}^{B} \partial_{B} Y_{2}^{A}-(1 \leftrightarrow 2), \\
\hat{\omega} & =0
\end{aligned}
$$

Now, we discuss the two relevant sub-cases of boundary conditions in asymptotically flat spacetimes. Adding the condition (3.16) to the preliminary condition (3.15), i.e. considering (AF1), gives the additional constraint

$$
\omega=0
$$

Note that this case corresponds exactly to the flat limit of the (AAdS1) case (equations (3.19) and (3.21)). The asymptotic symmetry algebra reduces to the semi-direct product

$$
\operatorname{Diff}\left(S^{2}\right) \ltimes \mathscr{S},
$$

where $\operatorname{Diff}\left(S^{2}\right)$ are the smooth superrotations generated by $Y^{A}$ and $\mathscr{S}$ are the smooth supertranslations generated by $T$. This extension of the original global $\mathrm{BMS}_{4}$ algebra (see below) is called the generalized $B M S_{4}$ algebra $[51,52,65,94]$. Therefore, the $\Lambda-\mathrm{BMS}_{4}$ algebra reduces in the flat limit to the smooth extension (3.69) of the $\mathrm{BMS}_{4}$ algebra.

The other sub-case of boundary conditions for asymptotically flat spacetimes (AF2) is given by adding condition (3.17) to the preliminary boundary condition (3.15). The additional constraint on the parameters is now given by

$$
D_{A} Y_{B}+D_{B} Y_{A}=D_{C} Y^{C} \stackrel{\circ}{q B}_{A B},
$$

i.e. $Y^{A}$ is a conformal Killing vector of the unit round sphere metric $\stackrel{\circ}{A B}_{A B}$. If we allow $Y^{A}$ to not be globally well-defined on the 2 -sphere, then the asymptotic symmetry algebra has the structure

$$
\left(\operatorname{Vir} \times \operatorname{Vir} \ltimes \mathscr{S}^{*}\right) \times \mathbb{R} .
$$

Here, Vir $\times$ Vir is the direct product of two copies of the Witt algebra, parametrized by $Y^{A}$. Furthermore, $\mathscr{S}^{*}$ are the supertranslations, parametrized by $T$, and $\mathbb{R}$ are the abelian Weyl rescalings of $\stackrel{\circ}{q}_{A B}$, parametrized by $\omega$. Note that the supetranslations also contain singular elements since they are related to the singular superrotations through the algebra (3.67). This extension of the global $\mathrm{BMS}_{4}$ algebra is called the extended BMS ${ }_{4}$ algebra [36]. Finally, as a sub-case of this one, considering the more restrictive constraints (3.18), i.e. (AF3), and allowing only globally well-defined $Y^{A}$, we recover the global $B M S_{4}$ algebra $[43,154,155]$, which is given by

$$
S O(3,1) \ltimes \mathscr{S},
$$

where $\mathscr{S}$ are the supertranslations and $S O(3,1)$ is the algebra of the globally well-defined conformal Killing vectors of the unit 2-sphere metric, which is isomorphic to the proper orthocronous Lorentz group in four dimensions. 
Definition [Action on the solution space] Given boundary conditions imposed in a chosen gauge, there is a natural action of the asymptotic symmetry algebra, with generators $F=F(a)$, on the solution space $\tilde{\Phi}=\tilde{\Phi}(b)$. The form of this action can be deduced from (3.2) by inserting the solution space and the explicit form of the asymptotic symmetry generators ${ }^{8}$.

Examples In the Fefferman-Graham gauge with Dirichlet boundary conditions for asymptotically $\mathrm{AdS}_{4}$ spacetimes (AAdS2) ((3.19) with (3.23)), the asymptotic symmetry algebra $S O(3,2)$ acts on the solution space (3.32) as

$$
\delta_{\xi_{0}} T_{a b}=\left(\mathscr{L}_{\xi_{0}^{c}}+\frac{1}{3} D_{c}^{(0)} \xi_{0}^{c}\right) T_{a b}
$$

In the Bondi gauge with definition (AF3) ((3.15) with (3.18)) of asymptotically flat spacetime, the global $\mathrm{BMS}_{4}$ algebra acts on the leading functions of the solution space (3.52) as

$$
\begin{aligned}
\delta_{(f, Y)} C_{A B}= & {\left[f \partial_{u}+\mathscr{L}_{Y}-\frac{1}{2} D_{C} Y^{C}\right] C_{A B}-2 D_{A} D_{B} f+\stackrel{\circ}{A B}_{A B} D_{C} D^{C} f, } \\
\delta_{(f, Y)} M= & {\left[f \partial_{u}+\mathscr{L}_{Y}+\frac{3}{2} D_{C} Y^{C}\right] M+\frac{1}{4} N^{A B} D_{A} D_{B} f+\frac{1}{2} D_{A} f D_{B} N^{A B}+\frac{1}{8} D_{C} D_{B} D_{A} Y^{A} C^{B C}, } \\
\delta_{(f, Y)} N_{A}= & {\left[f \partial_{u}+\mathscr{L}_{Y}+D_{C} Y^{C}\right] N_{A}+3 M D_{A} f-\frac{3}{16} D_{A} f N_{B C} C^{B C}+\frac{1}{2} D_{B} f N^{B C} C_{A C} } \\
& -\frac{1}{32} D_{A} D_{B} Y^{B} C_{C D} C^{C D}+\frac{1}{4}\left(2 D^{B} f+D^{B} D_{C} D^{C} f\right) C_{A B} \\
& -\frac{3}{4} D_{B} f\left(D^{B} D^{C} C_{A C}-D_{A} D_{C} C^{B C}\right)+\frac{3}{8} D_{A}\left(D_{C} D_{B} f C^{B C}\right) \\
& +\frac{1}{2}\left(D_{A} D_{B} f-\frac{1}{2} D_{C} D^{C} f \stackrel{\circ}{A B}_{A B}\right) D_{C} C^{B C},
\end{aligned}
$$

where $N_{A B}=\partial_{u} C_{A B}$ [36]. For the action of the associated asymptotic symmetry group on these solution spaces, see [35].

\section{Surface charges}

In this section, we review how to construct the surface charges associated with gauge symmetries. After recalling some results about global symmetries and Noether currents, the BarnichBrandt prescription to obtain the surface charges in the context of asymptotic symmetries is discussed. We illustrate this construction with the example of general relativity with asymptotically (A)dS and asymptotically flat spacetimes. The relation between this prescription and the covariant phase space methods is established.

\subsection{Global symmetries and Noether's first theorem}

Definition [Global symmetry] Let us consider a Lagrangian theory with Lagrangian density $\mathbf{L}\left[\Phi, \partial_{\mu} \Phi, \ldots\right]$ and a transformation $\delta_{Q} \Phi=Q$ of the fields, where $Q$ is a local function. In agreement with the above definition (3.3), this transformation is said to be a symmetry of the theory if

$$
\delta_{Q} \mathbf{L}=\mathrm{d} \mathbf{B}_{Q},
$$

\footnotetext{
${ }^{8}$ This action is usually not linear. However, in three-dimensional general relativity, this action is precisely the coadjoint representation of the asymptotic symmetry algebra [27, 28, 33, 34, 138].
} 
where $\mathbf{B}_{Q}=B_{Q}^{\mu}\left(\mathrm{d}^{n-1} x\right)_{\mu}$. Then, as defined in (3.2), a gauge symmetry is just a symmetry that depends on arbitrary spacetime functions $F=\left(f^{\alpha}\right)$, i.e. $Q=R[F]$. We define an on-shell equivalence relation $\sim$ between the symmetries of the theory as

$$
Q \sim Q+R[F],
$$

i.e. two symmetries are equivalent if they differ, on-shell, by a gauge transformation $R[F]$. The equivalence classes $[Q]$ for this equivalence relation are called the global symmetries. In particular, a gauge symmetry is a trivial global symmetry.

Definition [Noether current] A conserved current $\mathbf{j}$ is an on-shell closed $(n-1)$-form, i.e. $\mathrm{d} \mathbf{j} \approx 0$. We define an on-shell equivalence relation $\sim$ between the currents as

$$
\mathbf{j} \sim \mathbf{j}+\mathrm{d} \mathbf{K},
$$

where $\mathbf{K}$ is a $(n-2)$-form. A Noether current is an equivalence class $[\mathbf{j}]$ for this equivalence relation.

Theorem [Noether's first theorem] A one-to-one correspondence exists between global symmetries $Q$ and Noether currents $[\mathbf{j}]$, which can be written as

$$
[Q] \stackrel{1-1}{\longleftrightarrow}[\mathbf{j}] .
$$

In particular, Noether currents associated with gauge symmetries are trivial. Recent demonstrations of this theorem can for example be found in $[22,26]$.

Remark This theorem also enables us to construct explicit representatives of the Noether current for a given global symmetry. We have

$$
\delta_{Q} \mathbf{L}=\mathrm{d} \mathbf{B}_{Q}=\left(\partial_{\mu} B_{Q}^{\mu}\right) \mathrm{d}^{n} x .
$$

Furthermore, writing $\mathbf{L}=L \mathrm{~d}^{n} x$, we obtain

$$
\begin{aligned}
\delta_{Q} L & =\delta_{Q} \Phi \frac{\partial L}{\partial \Phi}+\delta_{Q} \partial_{\mu} \Phi \frac{\partial L}{\partial\left(\partial_{\mu} \Phi\right)}+\ldots \\
& =Q \frac{\partial L}{\partial \Phi}+\partial_{\mu} Q \frac{\partial L}{\partial\left(\partial_{\mu} \Phi\right)}+\ldots \\
& =Q\left(\frac{\partial L}{\partial \Phi}-\partial_{\mu} \frac{\partial L}{\partial\left(\partial_{\mu} \Phi\right)}+\ldots\right)+\partial_{\mu}\left(Q \frac{\partial L}{\partial\left(\partial_{\mu} \Phi\right)}+\ldots\right) \\
& =Q \frac{\delta L}{\delta \Phi}+\partial_{\mu}\left(Q \frac{\partial L}{\partial\left(\partial_{\mu} \Phi\right)}+\ldots\right),
\end{aligned}
$$

where, in the second line, we used

$$
\left[\delta_{Q}, \partial_{\mu}\right]=0
$$

and, in the last equality, we used (B.5). Putting (4.5) and (4.6) together, we obtain

$$
Q \frac{\delta L}{\delta \Phi}=\partial_{\mu}\left(B_{Q}^{\mu}-Q \frac{\partial L}{\partial\left(\partial_{\mu} \Phi\right)}+\ldots\right) \equiv \partial_{\mu} j_{Q}^{\mu}
$$


or, equivalently

$$
Q \frac{\delta \mathbf{L}}{\delta \Phi}=\mathrm{d}_{Q}
$$

where $\mathbf{j}_{Q}=j_{Q}^{\mu}\left(\mathrm{d}^{n-1} x\right)_{\mu}$. In particular, $\mathbf{d}_{Q} \approx 0$ holds on-shell. Hence, we have obtained a representative of the Noether current associated with the global symmetry $Q$ through the correspondence (4.4).

Theorem [Noether representation theorem] Defining the bracket as

$$
\left\{\mathbf{j}_{Q_{1}}, \mathbf{j}_{Q_{2}}\right\}=\delta_{Q_{1}} \mathbf{j}_{Q_{2}},
$$

we have

$$
\left\{\mathbf{j}_{Q_{1}}, \mathbf{j}_{Q_{2}}\right\} \approx \mathbf{j}_{\left[Q_{1}, Q_{2}\right]}
$$

( $n>1)$, where $\left[Q_{1}, Q_{2}\right]=\delta_{Q_{1}} Q_{2}-\delta_{Q_{2}} Q_{1}$. In other words, the Noether currents form a representation of the symmetries.

To prove this theorem, we apply $\delta_{Q_{1}}$ on the left-hand side and the right-hand side of (4.9), where $Q$ is replaced by $Q_{2}$. On the right-hand side, using the first equation of (B.8), we obtain

$$
\delta_{Q_{1}} \mathrm{~d} \mathbf{j}_{Q_{2}} \approx \mathrm{d} \delta_{Q_{1}} \mathbf{j}_{Q_{2}} .
$$

On the left-hand side, we have

$$
\begin{aligned}
\delta_{Q_{1}}\left(Q_{2} \frac{\delta \mathbf{L}}{\delta \Phi}\right) & =\delta_{Q_{1}} Q_{2} \frac{\delta \mathbf{L}}{\delta \Phi}+Q_{2} \delta_{Q_{1}} \frac{\delta \mathbf{L}}{\delta \Phi} \\
& =\delta_{Q_{1}} Q_{2} \frac{\delta \mathbf{L}}{\delta \Phi}+Q_{2} \frac{\delta}{\delta \Phi}\left(\delta_{Q_{1}} \mathbf{L}\right)-Q_{2} \sum_{k \geq 0}(-1)^{k} \partial_{\mu_{1}} \ldots \partial_{\mu_{k}}\left(\frac{\partial Q_{1}}{\partial \Phi_{\mu_{1} \ldots \mu_{k}}} \frac{\delta \mathbf{L}}{\delta \Phi}\right) \\
& =\delta_{Q_{1}} Q_{2} \frac{\delta \mathbf{L}}{\delta \Phi}-Q_{2} \sum_{k \geq 0}(-1)^{k} \partial_{\mu_{1}} \ldots \partial_{\mu_{k}}\left(\frac{\partial Q_{1}}{\partial \Phi_{\mu_{1} \ldots \mu_{k}}} \frac{\delta \mathbf{L}}{\delta \Phi}\right)
\end{aligned}
$$

where, to obtain the second equality, we used (B.9). In the last equality, we used (4.1) together with (B.6). Now, using Leibniz rules in the second term of the right-hand side, we get

$$
\begin{aligned}
\delta_{Q_{1}}\left(Q_{2} \frac{\delta \mathbf{L}}{\delta \Phi}\right) & =\delta_{Q_{1}} Q_{2} \frac{\delta \mathbf{L}}{\delta \Phi}-\sum_{k \geq 0} \partial_{\mu_{1}} \ldots \partial_{\mu_{k}} Q_{2}\left(\frac{\partial Q_{1}}{\partial \Phi_{\mu_{1} \ldots \mu_{k}}} \frac{\delta \mathbf{L}}{\delta \Phi}\right)+\partial_{\mu} T_{Q_{1}}^{\mu}\left(Q_{2}, \frac{\delta L}{\delta \Phi}\right) \mathrm{d}^{n} x \\
& =\left(\delta_{Q_{1}} Q_{2}-\delta_{Q_{2}} Q_{1}\right) \frac{\delta \mathbf{L}}{\delta \Phi}+\partial_{\mu} T_{Q_{1}}^{\mu}\left(Q_{2}, \frac{\delta L}{\delta \Phi}\right) \mathrm{d}^{n} x \\
& =\left[Q_{1}, Q_{2}\right] \frac{\delta \mathbf{L}}{\delta \Phi}+\partial_{\mu} T_{Q_{1}}^{\mu}\left(Q_{2}, \frac{\delta L}{\delta \Phi}\right) d^{n} x \\
& =\mathrm{dj}_{\left[Q_{1}, Q_{2}\right]}+\mathrm{d} \mathbf{T}_{Q_{1}}\left(Q_{2}, \frac{\delta L}{\delta \Phi}\right)
\end{aligned}
$$

where $T_{Q_{1}}^{\mu}\left(Q_{2}, \frac{\delta L}{\delta \Phi}\right)$ is an expression vanishing on-shell. In the second equality, we used (B.7), and in the last equality, we used (4.9). Putting (4.12) and (4.14) together results in

$$
\mathrm{d}\left[\delta_{Q_{1}} \mathbf{j}_{Q_{2}}-\mathbf{j}\left[Q_{1}, Q_{2}\right]-\mathbf{T}_{Q_{1}}\left(Q_{2}, \frac{\delta L}{\delta \Phi}\right)\right]=0
$$


We know from Poincaré lemma that locally, every closed form is exact ${ }^{9}$. However, this cannot be the case in Lagrangian field theories. In fact, this would imply that every $n$-form is exact, and therefore, there would not be any possibility of non-trivial dynamics. Let us remark that the operator $\mathrm{d}$ that we are using is not the standard exterior derivative, but a horizontal derivative in the jet bundle (see definition (B.3)) that takes into account the field-dependence. In this context, we have to use the algebraic Poincaré lemma.

Lemma [Algebraic Poincaré lemma] The cohomology class $H^{p}(\mathrm{~d})$ for the operator d defined in (B.3) is given by

$$
H^{p}(\mathrm{~d})= \begin{cases}{\left[\alpha^{n}\right]} & \text { if } p=n \\ 0 & \text { if } 0<p<n \\ \mathbb{R} & \text { if } p=0\end{cases}
$$

where $\left[\alpha^{n}\right]$ designates the equivalence classes of $n$-forms for the equivalence relation $\alpha^{n} \sim \alpha^{\prime n}$ if $\alpha^{n}=\alpha^{\prime n}+\mathrm{d} \beta^{n-1}[26]$.

Le us go back to the proof of (4.11). Applying the algebraic Poincaré lemma to (4.15) yields

$$
\delta_{Q_{1}} \mathbf{j}_{Q_{2}}=\mathbf{j}_{\left[Q_{1}, Q_{2}\right]}+\mathbf{T}_{Q_{1}}\left(Q_{2}, \frac{\delta L}{\delta \Phi}\right)+\mathrm{d} \eta
$$

where $\eta$ is a $(n-2)$-form. Therefore, on-shell, since $\mathbf{T}_{Q_{1}}\left(Q_{2}, \frac{\delta L}{\delta \Phi}\right) \approx 0$ and because Noether currents are defined up to exact $(n-1)$-forms, we obtain the result (4.11). Notice that in classical mechanics (i.e. $n=1$ ), from (4.16), constant central extensions may appear in the current algebra.

Definition [Noether charge] Given a Noether current [j] , we can construct a Noether charge by integrating it on a $(n-1)$-dimensional spacelike surface $\Sigma$, with boundary $\partial \Sigma$, as

$$
H_{Q}[\Phi]=\int_{\Sigma} \mathbf{j}
$$

If we assume that the currents and their ambiguities vanish at infinity, this definition does not depend on the representative of the Noether current. Indeed,

$$
H_{Q}^{\prime}[\Phi]=\int_{\Sigma}(\mathbf{j}+\mathrm{d} \mathbf{K})=H_{Q}[\Phi]+\int_{\partial \Sigma} \mathbf{K},
$$

where we used the Stokes theorem. Since $\int_{\partial \Sigma} \mathbf{K}=0$, we have $H_{Q}^{\prime}[\Phi]=H_{Q}[\Phi]$.

Remark [Conservation and algebra of Noether charges] The Noether charge (4.18) is conserved in time, that is,

$$
\frac{d}{d t} H_{Q}[\Phi] \approx 0
$$

${ }^{9}$ The Poincaré lemma states that in a star-shaped open subset, the de Rham cohomology class $H_{d R}^{p}$ is given by

$$
H_{d R}^{p}=\left\{\begin{array}{l}
0 \text { if } 0<p \leq n \\
\mathbb{R} \text { if } p=0
\end{array} .\right.
$$


In fact, consider two spacelike hypersurfaces $\Sigma_{1} \equiv t_{1}=0$ and $\Sigma_{2} \equiv t_{2}=0$. We have

$$
H_{Q}^{t_{2}}[\Phi]-H_{Q}^{t_{1}}[\Phi]=\int_{\Sigma_{2}} \mathbf{j}_{Q}-\int_{\Sigma_{1}} \mathbf{j}_{Q}=\int_{\Sigma_{2}-\Sigma_{1}} \mathrm{~d} \mathbf{j}_{Q} \approx 0
$$

where $\Sigma_{2}-\Sigma_{1}$ is the spacetime volume encompassed between $\Sigma_{1}$ and $\Sigma_{2}$. In the second equality, we used the hypothesis that currents vanish at infinity and the Stokes theorem.

The Noether charges (4.18) form a representation of the algebra of global symmetries, i.e.

$$
\left\{H_{Q_{1}}, H_{Q_{2}}\right\} \approx H_{\left[Q_{1}, Q_{2}\right]},
$$

where the bracket of Noether charges is defined as

$$
\left\{H_{Q_{1}}, H_{Q_{2}}\right\}=\delta_{Q_{1}} H_{Q_{2}}=\int_{\Sigma} \delta_{Q_{1}} \mathbf{j}_{Q_{2}}
$$

This is a direct consequence of (4.11).

\subsection{Gauge symmetries and lower degree conservation law}

Definition [Noether identities] Consider the relation (4.9) for a gauge symmetry:

$$
R[F] \frac{\delta L}{\delta \Phi}=\partial_{\mu} j_{F}^{\mu}
$$

The left-hand side can be worked out as

$$
\begin{aligned}
R[F] \frac{\delta L}{\delta \Phi}= & \left(R_{\alpha} f^{\alpha}+R_{\alpha}^{\mu} \partial_{\mu} f^{\alpha}+R_{\alpha}^{(\mu v)} \partial_{\mu} \partial_{v} f^{\alpha}+\ldots\right) \frac{\delta L}{\delta \Phi} \\
= & f^{\alpha}\left[R_{\alpha} \frac{\delta L}{\delta \Phi}-\partial_{\mu}\left(R_{\alpha}^{\mu} \frac{\delta L}{\delta \Phi}\right)+\partial_{\mu} \partial_{v}\left(R_{\alpha}^{(\mu v)} \frac{\delta L}{\delta \Phi}\right)+\ldots\right] \\
& +\partial_{\mu} \underbrace{\left[R_{\alpha}^{\mu} f^{\alpha} \frac{\delta L}{\delta \Phi}-f^{\alpha} \partial_{v}\left(R_{\alpha}^{(\mu v)} \frac{\delta L}{\delta \Phi}\right)+\ldots\right]}_{\equiv S_{F}^{\mu}} .
\end{aligned}
$$

Therefore, the equation (4.24) can be rewritten as

$$
f^{\alpha} R_{\alpha}^{\dagger}\left(\frac{\delta L}{\delta \Phi}\right)=\partial_{\mu}\left(j_{F}^{\mu}-S_{F}^{\mu}\right)
$$

where $R_{\alpha}^{\dagger}\left(\frac{\delta L}{\delta \Phi}\right)=R_{\alpha} \frac{\delta L}{\delta \Phi}-\partial_{\mu}\left(R_{\alpha}^{\mu} \frac{\delta L}{\delta \Phi}\right)+\partial_{\mu} \partial_{v}\left(R_{\alpha}^{(\mu v)} \frac{\delta L}{\delta \Phi}\right)+\ldots$ Since $F$ is a set of arbitrary functions, we can apply the Euler-Lagrange derivative (B.5) with respect to $f^{\alpha}$ on this equation. Since the right-hand side is a total derivative, it vanishes under the action of the Euler-Lagrange derivative (see (B.6)) and we obtain

$$
R_{\alpha}^{\dagger}\left(\frac{\delta \mathbf{L}}{\delta \Phi}\right)=0
$$

This identity is called a Noether identity. There is one identity for each independent generator $f^{\alpha}$. Notice that these identities are satisfied off-shell. 
Theorem [Noether's second theorem] We have

$$
R[F] \frac{\delta \mathbf{L}}{\delta \Phi}=\mathrm{d} \mathbf{S}_{F}\left[\frac{\delta L}{\delta \Phi}\right]
$$

where $\mathbf{S}_{F}=S_{F}^{\mu}\left(\mathrm{d}^{n-1} x\right)_{\mu}$ is the weakly vanishing Noether current (i.e. $\mathbf{S}_{F} \approx 0$ ) that was defined in the last line of (4.25). This is a direct consequence of (4.25), taking the Noether identity (4.27) into account.

Example Consider the theory of general relativity $\mathbf{L}=(16 \pi G)^{-1}(R-2 \Lambda) \sqrt{-g} \mathrm{~d}^{n} x$. The EulerLagrange derivative of the Lagrangian is given by

$$
\frac{\delta \mathbf{L}}{\delta g_{\mu v}}=-(16 \pi G)^{-1}\left(G^{\mu v}+g^{\mu v} \Lambda\right) \sqrt{-g} \mathrm{~d}^{n} x .
$$

The Noether identity associated with the diffeomorphism generated by $\xi^{\mu}$ is obtained by following the lines of (4.25):

$$
\begin{aligned}
(16 \pi G) \delta_{\xi} g_{\mu v} \frac{\delta \mathbf{L}}{\delta g_{\mu v}} & =-2 \nabla_{\mu} \xi_{v}\left(G^{\mu v}+g^{\mu v} \Lambda\right) \sqrt{-g} \mathrm{~d}^{n} x \\
& =2 \xi_{v} \nabla_{\mu} G^{\mu v} \sqrt{-g} \mathrm{~d}^{n} x-\partial_{\mu}\left[2 \xi_{v}\left(G^{\mu v}+g^{\mu v} \Lambda\right) \sqrt{-g}\right] \mathrm{d}^{n} x
\end{aligned}
$$

Therefore, the Noether identity is the Bianchi identity for the Einstein tensor

$$
\nabla_{\mu} G^{\mu v}=0
$$

and the weakly vanishing Noether current of Noether's second theorem (4.28) is given by

$$
\mathbf{S}_{\xi}=-\frac{\sqrt{-g}}{8 \pi G} \xi_{v}\left(G^{\mu v}+g^{\mu v} \Lambda\right)\left(\mathrm{d}^{n-1} x\right)_{\mu}
$$

Remark From (4.24) and (4.28), we have $\mathrm{d}\left(\mathbf{j}_{F}-\mathbf{S}_{F}\right)=0$, and hence, from the algebraic Poincaré lemma (4.16),

$$
\mathbf{j}_{F}=\mathbf{S}_{F}+\mathrm{d} \mathbf{K}_{F},
$$

where $\mathbf{K}_{F}$ is a $(n-2)$-form. Therefore, as already stated in Noether's first theorem (4.4), the Noether current associated with a gauge symmetry is trivial, i.e. vanishing on-shell, up to an exact $(n-1)$-form. A natural question arises at this stage: is it possible to define a notion of conserved quantity for gauge symmetries? Naively, following the definition (4.18), one may propose the following definition for conserved charge:

$$
H_{F}=\int_{\Sigma} \mathbf{j}_{F} \approx \int_{\partial \Sigma} \mathbf{K}_{F}
$$

where, in the second equality, we used (4.33) and Stokes' theorem. This charge will be conserved on-shell since $\mathrm{d} \mathbf{j}_{F} \approx 0$. The problem is that the $(n-2)$-form $\mathbf{K}_{F}$ appearing in (4.34) is completely arbitrary. Indeed, the Noether currents are equivalence classes of currents (see equation (4.3)). Therefore, we have to find an appropriate procedure to isolate a particular $\mathbf{K}_{F}$. 
Definition [Reducibility parameter] Reducibility parameters $\bar{F}$ are parameters of gauge transformations satisfying

$$
R[\bar{F}] \approx 0
$$

Two reducibility parameters $\bar{F}$ and $\bar{F}^{\prime}$ are said to be equivalent, i.e. $\bar{F} \sim \bar{F}^{\prime}$, if $\bar{F} \approx \bar{F}^{\prime}$. Note that for a large class of gauge theories (including electrodynamics, Yang-Mills and general relativity in dimensions superior or equal to three $[22,26]$ ), these equivalence classes of asymptotic reducibility parameters are determined by field-independent ordinary functions $\bar{F}(x)$ satisfying the off-shell condition

$$
R[\bar{F}]=0 .
$$

We will call them exact reducibility parameters.

Theorem [Generalized Noether's theorem] A one-to-one correspondence exists between equivalence classes of reducibility parameters and equivalence classes of on-shell conserved $(n-2)$ forms $[\mathbf{K}]$, which can be written as

$$
[\bar{F}] \stackrel{1-1}{\longleftrightarrow}[\mathbf{K}] .
$$

In this statement, two conserved ( $n-2)$-forms $\mathbf{K}$ and $\mathbf{K}^{\prime}$ are said to be equivalent, i.e. $\mathbf{K} \sim \mathbf{K}^{\prime}$, if $\mathbf{K} \approx \mathbf{K}^{\prime}+\mathrm{d} \mathbf{l}$ where $\mathbf{l}$ is a $(n-3)$-form [23, 24].

Remark The Barnich-Brandt procedure allows for the construction of explicit representatives of the conserved $(n-2)$-forms for given exact reducibility parameters $\bar{F}$ [18, 22]. From Noether's second theorem (4.28) and (4.36), we have

$$
\mathrm{d} \mathbf{S}_{\bar{F}}=0 .
$$

From the algebraic Poincaré Lemma (4.16), we get $^{10}$

$$
-\mathrm{d} \mathbf{K}_{\bar{F}}=\mathbf{S}_{\bar{F}} \approx 0 \text {. }
$$

Using the homotopy operator (B.16), we define

$$
\mathbf{k}_{\bar{F}}[\Phi ; \delta \Phi]=-I_{\delta \Phi}^{n-1} \mathbf{S}_{\bar{F}} .
$$

This $\mathbf{k}_{\bar{F}}[\Phi ; \delta \Phi]$ is an element of $\Omega^{n-2,1}$ (see appendix B) and is defined up to an exact $(n-2)$-form. This enables us to find an explicit expression for the conserved $(n-2)$-form $\mathbf{K}_{\bar{F}}[\Phi]$ as

$$
\mathbf{K}_{\bar{F}}[\Phi]=\int_{\gamma} \mathbf{k}_{\bar{F}}[\Phi ; \delta \Phi]
$$

where $\gamma$ is a path on the solution space relating $\bar{\Phi}$ such that $S_{\bar{F}}[\bar{\Phi}]=0$ to the solution $\Phi$ of interest. Applying the operator $d$ on (4.41) gives back (4.39), using the property (B.18) of the homotopy operator. Notice that the expression (4.41) of $\mathbf{K}_{\bar{F}}[\Phi]$ generically depends on the chosen path $\gamma$. Therefore, in practice, we consider the $(n-2)$-form $\mathbf{k}_{\bar{F}}[\Phi ; \delta \Phi]$ defined in (4.40) as the fundamental object, rather than $\mathbf{K}_{\bar{F}}[\Phi]$.

\footnotetext{
${ }^{10}$ The minus sign on the left-hand side of (4.39) is a matter of convention.
} 
Example Let us return to our example of general relativity. The exact reducibility parameters of the theory are the diffeomorphism generators $\bar{\xi}$, which satisfy

$$
\delta_{\bar{\xi}} g_{\mu \nu}=\mathscr{L}_{\bar{\xi}} g_{\mu \nu}=0
$$

i.e. they are the Killing vectors of $g_{\mu \nu}$. Note that for a generic metric, this equation does not admit any solution. Hence, the previous construction is irrelevant for this general case. Now, consider linearized general relativity around a background $g_{\mu \nu}=\bar{g}_{\mu \nu}+h_{\mu \nu}$. We can show that

$$
\delta_{\bar{\xi}} h_{\mu v}=\mathscr{L}_{\bar{\xi}} \bar{g}_{\mu v}=0
$$

i.e. the exact reducibility parameters of the linearized theory are the Killing vectors of the background $\bar{g}_{\mu \nu}$. If $\bar{g}_{\mu \nu}$ is taken to be the Minkowski metric, then the solutions of (4.43) are the generators of the Poincaré transformations. The (n-2)-form (4.41) can be constructed explicitly and integrated on a $(n-2)$-sphere at infinity. This gives the ADM charges of linearized gravity [22].

\subsection{Asymptotic symmetries and surface charges}

We now come to the case of main interest, where we are dealing with asymptotic symmetries in the sense of the definition in subsection 3.4. The prescription to construct the $(n-2)$-form $\mathbf{k}_{F}[\Phi, \delta \Phi]$ associated with generators of asymptotic symmetries $F$ is essentially the same as the one introduced above for exact reducibility parameters. However, this $(n-2)$-form will not be conserved on-shell. Indeed, for a generic asymptotic symmetry, (4.38) does not hold; therefore, the weak equality in (4.39) is not valid anymore. Nonetheless, as we will see below, we still have a control on the breaking in the conservation law.

Definition [Barnich-Brandt $(n-2)$-form for asymptotic symmetries] The $(n-2)$-form $\mathbf{k}_{F}$ associated with asymptotic symmetries generated by $F$ is defined as

$$
\mathbf{k}_{F}[\Phi ; \delta \Phi]=-I_{\delta \Phi}^{n-1} \mathbf{S}_{F}
$$

where $I_{\delta \Phi}^{n-1}$ is the homotopy operator (B.16) and $\mathbf{S}_{F}$ is the weakly vanishing Noether current defined in the last line of (4.25). For a first order gauge theory, namely a gauge theory involving only first order derivatives of the gauge parameters $F=\left(f^{\alpha}\right)$ in the gauge transformations as in (3.6) and first order equations of motion for the fields $\Phi=\left(\phi^{i}\right)$, the $(n-2)$-form (4.44) becomes

$$
\mathbf{k}_{F}[\Phi ; \delta \Phi]=-\frac{1}{2} \delta \Phi \frac{\partial}{\partial\left(\partial_{\mu} \Phi\right)}\left(\frac{\partial}{\partial \mathrm{d} x^{\mu}} \mathbf{S}_{F}\right)
$$

where

$$
\mathbf{S}_{F}=R_{\alpha}^{\mu} f^{\alpha} \frac{\delta L}{\delta \Phi}\left(\mathrm{d}^{n-1} x\right)_{\mu}
$$

The simplicity of these expressions motivates the study of first order formulations of gauge theories in this context $[31,32,95]$. 
Example Let us consider the theory of general relativity. Applying the homotopy operator on the weakly vanishing Noether current $\mathbf{S}_{\xi}$ obtained in equation (4.32), we deduce the explicit expression

$$
\begin{aligned}
\mathbf{k}_{\xi}[g ; h]=\frac{\sqrt{-g}}{8 \pi G}\left(\mathrm{~d}^{n-2} x\right)_{\mu v}\left[\xi^{v} \nabla^{\mu} h+\xi^{\mu} \nabla_{\sigma} h^{\sigma v}+\xi_{\sigma} \nabla^{v} h^{\sigma \mu}\right. \\
\left.+\frac{1}{2}\left(h \nabla^{v} \xi^{\mu}+h^{\mu \sigma} \nabla_{\sigma} \xi^{v}+h^{v \sigma} \nabla^{\mu} \xi_{\sigma}\right)\right],
\end{aligned}
$$

where $h_{\mu \nu}=\delta g_{\mu \nu}$. Indices are lowered and raised by $g_{\mu \nu}$ and its inverse, and $h=h^{\mu}{ }_{\mu}$ [22]. Notice that this expression has also been derived in the first order Cartan formulation of general relativity in [31] (see also [32]).

Theorem [Conservation law] Define the invariant presymplectic current as

$$
\mathbf{W}[\Phi ; \delta \Phi, \delta \Phi]=\frac{1}{2} I_{\delta \Phi}^{n}\left(\delta \Phi \frac{\delta \mathbf{L}}{\delta \Phi}\right) .
$$

We have the following conservation law

$$
\mathrm{dk}_{F}[\Phi ; \delta \Phi] \approx \mathbf{W}[\Phi ; R[F], \delta \Phi]
$$

where, in the equality $\approx$, it is implied that $\Phi$ is a solution of the Euler-Lagrange equations and $\delta \Phi$ is a solution of the linearized Euler-Lagrange equations. Furthermore, we use the notation $\mathbf{W}[\Phi ; R[F], \delta \Phi]=i_{R[F]} \mathbf{W}[\Phi ; \delta \Phi, \delta \Phi]$.

The proof of this proposition involves the properties of the operators introduced in appendix B. We have

$$
\begin{aligned}
\mathrm{d}_{F}[\Phi ; \delta \Phi] & =-\mathrm{d} I_{\delta \Phi}^{n-1} \mathbf{S}_{F} \\
& =\delta \mathbf{S}_{F}-I_{\delta \Phi}^{n} \mathrm{~d} \mathbf{S}_{F} \\
& \approx-I_{\delta \Phi}^{n} \mathrm{~d} \mathbf{S}_{F} \\
& \approx-I_{\delta \Phi}^{n}\left(R[F] \frac{\delta \mathbf{L}}{\delta \Phi}\right) \\
& \approx \frac{1}{2} i_{R[F]} I_{\delta \Phi}^{n}\left(\delta \Phi \frac{\delta \mathbf{L}}{\delta \Phi}\right) \\
& \approx i_{R[F]} \mathbf{W}[\Phi ; \delta \Phi, \delta \Phi] \\
& \approx \mathbf{W}[\Phi ; R[F], \delta \Phi] .
\end{aligned}
$$

In the second equality, we used (B.18). In the third equality, we used the fact that $\delta \mathbf{S}_{F} \approx 0$, since $\delta \Phi$ is a solution of the linearized Euler-Lagrange equations. In the fourth equality, we used Noether's second theorem (4.28). In the fifth equality, we used

$$
i_{R[F]} \mathbf{W}[\Phi ; \delta \Phi, \delta \Phi]=I_{R[F]}^{n}\left(\delta \Phi \frac{\delta \mathbf{L}}{\delta \Phi}\right)=-I_{\delta \Phi}^{n}\left(R[F] \frac{\delta \mathbf{L}}{\delta \Phi}\right) .
$$

The proof of this statement can be found in appendix A.5 of [25]. Finally, in the sixth equality, we used the definition (4.48). 
Definition [Surface charges] Let $\Sigma$ be a $(n-1)$-surface and $\partial \Sigma$ its $(n-2)$-dimensional boundary. We define the infinitesimal surface charge $\phi H_{F}[\Phi]$ as

$$
\phi H_{F}[\Phi]=\int_{\partial \Sigma} \mathbf{k}_{F}[\Phi ; \delta \Phi] \approx \int_{\Sigma} \mathbf{W}[\Phi ; R[F], \delta \Phi] .
$$

The infinitesimal surface charge $\phi H_{F}[\Phi]$ is said to be integrable if it is $\delta$-exact, i.e. if $\phi H_{F}[\Phi]=\delta H_{F}[\Phi]$. The symbol $\phi$ in (4.52) emphasizes that the infinitesimal surface charge is not necessarily integrable. If it is actually integrable, then we can define the integrated surface charge $H_{F}[\Phi]$ as

$$
H_{F}[\Phi]=\int_{\gamma} \delta H_{F}[\Phi]+N[\bar{\Phi}]=\int_{\gamma} \int_{\partial \Sigma} \mathbf{k}_{F}[\Phi ; \delta \Phi]+N[\bar{\Phi}],
$$

where $\gamma$ is a path in the solution space, going from a reference solution $\bar{\Phi}$ to the solution $\Phi . N[\bar{\Phi}]$ is a chosen value of the charge for this reference solution, which is not fixed by the formalism. Notice that for integrable infinitesimal charge, the integrated charge $H_{F}[\Phi]$ is independent from the chosen path $\gamma$ [63].

Theorem [Charge representation theorem] Assuming integrability, the integrated surface charges satisfy the algebra

$$
\left\{H_{F_{1}}, H_{F_{2}}\right\} \approx H_{\left[F_{1}, F_{2}\right]_{A}}+K_{F_{1} ; F_{2}}[\bar{\Phi}] .
$$

In this expression, the integrated charges bracket is defined as

$$
\left\{H_{F_{1}}, H_{F_{2}}\right\}=\delta_{F_{2}} H_{F_{1}}=\int_{\partial \Sigma} \mathbf{k}_{F_{1}}\left[\Phi ; \delta_{F_{2}} \Phi\right] .
$$

Furthermore, the central extension $K_{F_{1} ; F_{2}}[\bar{\Phi}]$, which depends only on the reference solution $\bar{\Phi}$, is antisymmetric with respect to $F_{1}$ and $F_{2}$, i.e. $K_{F_{1} ; F_{2}}[\bar{\Phi}]=K_{F_{2} ; F_{1}}[\bar{\Phi}]$. It satisfies the 2-cocycle condition

$$
K_{\left[F_{1}, F_{2}\right]_{A} ; F_{3}}[\bar{\Phi}]+K_{\left[F_{2}, F_{3}\right]_{A} ; F_{1}}[\bar{\Phi}]+K_{\left[F_{3}, F_{1}\right]_{A} ; F_{2}}[\bar{\Phi}] \approx 0 .
$$

Therefore, the integrated charges form a representation of the asymptotic symmetry algebra, up to a central extension $[22,25]$.

For the proof of this theorem, see e.g. section 1.4 of [63].

Remark In the literature, there are several criteria based on properties of the surface charges, that make a choice of boundary conditions interesting. The main properties are the following:

- The charges are usually required to be finite. Two types of divergences may occur: divergences in the expansion parameter defining asymptotics, say $r$, and divergences when performing the integration on the $(n-2)$-surface $\partial \Sigma$.

- The charges have to be integrable. As explained above, this criterion enables us to define integrated surface charges as in (4.53). Integrability implies that the charges form a representation of the asymptotic symmetry algebra, up to a central extension (see (4.54)). Furthermore, the integrated charges generate the symmetries on the solution space. 
- The charges have to be generically non-vanishing. Indeed, since the integrated surface charges generate the symmetry, identically vanishing charges would imply trivial action on the solution space. In particular, the asymptotic symmetries for which associated integrated charges identically vanish are considered as trivial in the strong definition of asymptotic symmetry group (2.3).

- The charges have to be conserved in time when the integration is performed on a spacelike $(n-2)$-dimensional surface $\partial \Sigma$ at infinity. This statement is not guaranteed a priori because of the breaking in the conservation law (4.49).

However, even if these requirements seem reasonable, in practice, some of them may not be satisfied. Indeed, as we will see below, the BMS charges in four dimensions are not always finite, neither integrable, nor conserved [39]. We now discuss the violation of some of the above requirements:

- The fact that the charges may not be finite in terms of the expansion parameter $r$ can be expected when the asymptotic region is taken to be at infinity. Indeed, consider $r$ as a cutoff. It makes sense to integrate on a surface $\partial \Sigma$ at a constant finite value of $r$, encircling a finite volume. Then, taking the limit $r \rightarrow \infty$ leads to an infinite volume; therefore, it does not come as a surprise that quantities diverge. Furthermore, it has recently been shown in [97] that subleading orders in $r$ in the $(n-2)$-form $\mathbf{k}_{F}[\Phi ; \delta \Phi]$ contain some interesting physical information, such as the 10 conserved Newman-Penrose charges [133]. Therefore, it seems reasonable to think that overleading orders in $r$ may also contain relevant information (see e.g. $[64,70])$.

- The non-integrability of the charges may be circumvented by different procedures to isolate an integrable part in the expression of the charges (see e.g. [172] and [65]). However, the final integrated surface charges obtained by these procedures do not have all the properties that integrable charges would have. In particular, the representation theorem does not generically hold. Another philosophy is to keep working with non-integrable expressions, without making any specific choice for the integrable part of the charges. In some situations, it is still possible to define a modified bracket for the charges, leading to a representation of the asymptotic symmetry algebra, up to a 2-cocycle which may depend on fields [39,65]. However, no general representation theorem exists in this context, even if some progress has been made [170].

- Finally, the non-conservation of the charges contains some important information on the physics. For example, in asymptotically flat spacetimes at null infinity, the non-conservation in time of the charges associated with time translations is known as the Bondi mass loss. This tells us that the mass decreases in time at future null infinity because of a flux of radiation through the boundary. Hence, the non-conservation of the charges contains important information on the dynamics of the system.

Even if the charges have these pathologies, they still offer important insights on the system. They could be seen as interesting combinations of the elements of the solution space that enjoy some properties in their transformation (see e.g. [34,35]). 
Examples We now provide explicit examples of surface charge constructions in four-dimensional general relativity. First, consider asymptotically $\mathrm{AdS}_{4}$ spacetimes with Dirichlet boundary conditions (AAdS2) (condition (3.19) together with (3.23)), the associated solution derived in subsection 3.3 (equation (3.32)), and the associated asymptotic Killing vectors derived in subsection 3.4. Inserting this solution space and these asymptotic Killing vectors into the $(n-2)$-form (4.47) results in an integrable expression at order $\rho^{0}$. Therefore, we can construct an integrated surface charge (4.53) where the 2 -surface $\partial \Sigma$ is taken to be the 2 -sphere at infinity, written $S_{\infty}$. We have the explicit expression

$$
H_{\xi}[g]=\int_{S_{\infty}} \mathrm{d}^{2} \Omega\left(\xi_{0}^{a} T_{a}^{t}\right)
$$

where $\mathrm{d}^{2} \Omega$ is the integration measure on the 2-sphere (see e.g. [69]). These charges are finite and generically non-vanishing. Furthermore, we can easily show that they are conserved in time, i.e.

$$
\frac{d}{d t} H_{\xi}[g] \approx 0
$$

Now, we consider definition (3.15) with (3.18) of asymptotically flat spacetimes in four dimensions (AF3). The surface charges are obtained by inserting the corresponding solution space derived in subsection 3.3 (see equation (3.52)) and the asymptotic Killing vectors discussed in subsection 3.4 into the expression (4.47), and then integrating over $S_{\infty}$. The result is given by

$$
\phi H_{\xi}[g ; \delta g] \approx \delta J_{\xi}[g]+\Theta_{\xi}[g ; \delta g]
$$

where

$$
\begin{aligned}
& J_{\xi}[g]=\frac{1}{16 \pi G} \int_{S_{\infty}} \mathrm{d}^{2} \Omega\left[4 f M+Y^{A}\left(2 N_{A}+\frac{1}{16} \partial_{A}\left(C^{C B} C_{C B}\right)\right)\right] \\
& \Theta_{\xi}[g ; \delta g]=\frac{1}{16 \pi G} \int_{S_{\infty}} \mathrm{d}^{2} \Omega\left[\frac{f}{2} N_{A B} \delta C^{A B}\right]
\end{aligned}
$$

and where $N_{A B}=\partial_{u} C_{A B}$ [39]. As mentioned above, the infinitesimal surface charges are not integrable. Therefore, we cannot unambiguously define an integrated surface charge as in (4.53) (see, however, $[65,172])$. In particular, the representation theorem (4.54) does not hold. Nevertheless, we can define the following modified bracket [39]:

$$
\left\{J_{\xi_{1}}, J_{\xi_{2}}\right\}^{*}=\delta_{\xi_{2}} J_{\xi_{1}}[g]+\Theta_{\xi_{2}}\left[g ; \delta_{\xi_{1}} g\right]
$$

We can show that

$$
\left\{J_{\xi_{1}}, J_{\xi_{2}}\right\}^{*} \approx J_{\left[\xi_{1}, \xi_{2}\right]_{A}}[g]+K_{\xi_{1} ; \xi_{2}}[g]
$$

where $K_{\xi_{1} ; \xi_{2}}[g]$ is a field-dependent 2 -cocycle given explicitly by ${ }^{11}$

$$
K_{\xi_{1} ; \xi_{2}}[g]=\frac{1}{32 \pi G} \int_{S_{\infty}} \mathrm{d}^{2} \Omega\left[C^{B C}\left(f_{1} D_{B} D_{C} D_{A} Y_{2}^{A}-f_{2} D_{B} D_{C} D_{A} Y_{1}^{A}\right)\right] .
$$

\footnotetext{
${ }^{11}$ Notice that this 2-cocycle is zero for globally well-defined conformal transformations on the 2-sphere. It becomes non-trivial when considering the extended $\mathrm{BMS}_{4}$ group with $\operatorname{Vir} \times \operatorname{Vir}$ superrotations.
} 
It satisfies the generalized 2-cocycle condition

$$
K_{\left[\xi_{1}, \xi_{2}\right]_{A}, \xi_{3}}+\delta_{\xi_{3}} K_{\xi_{1}, \xi_{2}}+\operatorname{cyclic}(1,2,3) \approx 0
$$

For the algebra (4.62) to make sense, its form should not depend on the particular choice of integrable part $J_{\xi}[g]$ in (4.60). In particular, defining $J^{\prime}=J-N$ and $\Theta^{\prime}=\Theta+\delta N$ for some $N=N_{\xi}[g]$, we obtain

$$
\left\{J_{\xi_{1}}^{\prime}, J_{\xi_{2}}^{\prime}\right\}^{*}=J_{\left[\xi_{1}, \xi_{2}\right]_{A}}^{\prime}[g]+K_{\xi_{1} ; \xi_{2}}^{\prime}[g]
$$

where $\left\{J_{\xi_{1}}^{\prime}, J_{\xi_{2}}^{\prime}\right\}^{*}=\delta_{\xi_{2}} J_{\xi_{1}}^{\prime}[g]+\Theta_{\xi_{2}}^{\prime}\left[g ; \delta_{\xi_{1}} g\right]$ and

$$
K_{\xi_{1} ; \xi_{2}}^{\prime}=K_{\xi_{1}, \xi_{2}}-\delta_{\xi_{2}} N_{\xi_{1}}+\delta_{\xi_{1}} N_{\xi_{2}}+N_{\left[\xi_{1}, \xi_{2}\right]_{A}}
$$

Notice that $-\delta_{\xi_{2}} N_{\xi_{1}}+\delta_{\xi_{1}} N_{\xi_{2}}+N_{\left[\xi_{1}, \xi_{2}\right]_{A}}$ automatically satisfies the generalized 2-cocycle condition (4.64) [39]. Another property of the surface charges (4.59) and (4.60) is that they are not conserved. Indeed,

$$
\frac{d}{d u} \not H_{\xi}[g]=\int_{S_{\infty}} \mathbf{W}\left[g ; \delta_{\xi} g, \delta g\right]
$$

where $\mathbf{W}[g ; \delta g, \delta g]$ was computed ${ }^{12}$ in [65]. We have

$$
\int_{S_{\infty}} \mathbf{W}[g ; \delta g, \delta g]=-\frac{1}{32 \pi G} \int_{S_{\infty}} \mathrm{d}^{2} \Omega\left[\delta N^{A B} \wedge \delta C_{A B}\right]
$$

Notice that taking $f=1$ and $Y^{A}=0$ in (4.67), we recover the famous Bondi mass loss formula $[43,154,155]$. This formula indicates that the mass is decreasing in time because of the leak of radiation through $\mathscr{I}^{+}$. This was a striking argument for the existence of gravitational waves at the non-linear level of the theory. Finally, despite the BMS charges (4.59) and (4.60) not being divergent in $r$, we can show that some of the supertranslation charges diverge for the Kerr solution [39].

Remark A non-trivial relation seems to exist between conservation and integrability of the surface charges. For example, in the case of Dirichlet boundary conditions in asymptotically $\mathrm{AdS}_{4}$ spacetimes (AAdS2) considered above, we see that the surface charges are both integrable and conserved. Reciprocally, there is a relation between non-conservation and non-integrability of the surface charges. For example, in the asymptotically flat case (AF3), we see that the source of nonintegrability is contained in the asymptotic shear $C_{A B}$ and the news function $N_{A B}=\partial_{u} C_{A B}$. These are precisely the functions involved in the right-hand side of (4.68). We can consider many other examples where this phenomenon appears. Therefore, non-integrability is related to non-conservation of the charges. We will see below that for diffeomorphism-invariant theories, the relation between non-conservation and integrability is transparent in the covariant phase space formalism.

\footnotetext{
${ }^{12}$ More precisely, in [65], we computed the presymplectic potential $\omega[g ; \delta g, \delta g]$ introduced below. However, as we will see, this is equal to the invariant presymplectic current in the Bondi gauge.
} 


\subsection{Relation between Barnich-Brandt and Iyer-Wald procedures}

In this subsection, we briefly discuss the covariant phase space formalism leading to the IyerWald prescription for surface charges [104, 117, 171, 172]. Notice that this method is valid only for diffeomorphism-invariant theories (including general relativity), and not for any gauge theories. In practice, this means that the parameters of the asymptotic symmetries are diffeomorphisms generators, i.e. $F \equiv \xi$ and $\delta_{F} \Phi \equiv \mathscr{L}_{\xi} \Phi$. Finally, we relate this prescription to the Barnich-Brandt prescription presented in detail in the previous section.

Definition [Presymplectic form] Consider a diffeomorphism-invariant theory with Lagrangian $\mathbf{L}=L \mathrm{~d}^{n} x$. Let us perform an arbitrary variation of the Lagrangian. Using a similar procedure as in (4.6), we obtain

$$
\begin{aligned}
\delta L & =\delta \Phi \frac{\partial L}{\partial \Phi}+\delta \partial_{\mu} \Phi \frac{\partial L}{\partial\left(\partial_{\mu} \Phi\right)}+\ldots \\
& =\delta \Phi \frac{\delta L}{\delta \Phi}+\partial_{\mu}\left(\delta \Phi \frac{\partial L}{\partial\left(\partial_{\mu} \Phi\right)}+\ldots\right) \\
& =\delta \Phi \frac{\delta L}{\delta \Phi}+\partial_{\mu} \theta^{\mu}[\Phi ; \delta \Phi]
\end{aligned}
$$

where

$$
\theta[\Phi ; \delta \Phi]=\theta^{\mu}[\Phi ; \delta \Phi]\left(\mathrm{d}^{n-1} x\right)_{\mu}=\left(\delta \Phi \frac{\partial L}{\partial\left(\partial_{\mu} \Phi\right)}+\ldots\right)\left(\mathrm{d}^{n-1} x\right)_{\mu}=I_{\delta \Phi}^{n} \mathbf{L}
$$

is the presymplectic potential. Taking into account that $\delta$ is Grassmann odd, the equation (4.69) can be rewritten as

$$
\delta \mathbf{L}=\delta \Phi \frac{\delta \mathbf{L}}{\delta \Phi}-\mathrm{d} \theta[\Phi ; \delta \Phi]
$$

Now, the presymplectic form $\omega$ is defined as

$$
\omega[\Phi ; \delta \Phi, \delta \Phi]=\delta \theta[\Phi, \delta \Phi]
$$

Definition [Iyer-Wald $(n-2)$-form for asymptotic symmetries] The Iyer-Wald $(n-2)$-form $\mathbf{k}_{\xi}^{I W}$ associated with asymptotic symmetries generated by $\xi$ is defined as

$$
\mathbf{k}_{\xi}^{I W}[\Phi ; \delta \Phi]=-\delta \mathbf{Q}_{\xi}[\Phi]+\iota_{\xi} \theta[\Phi ; \delta \Phi],
$$

up to an exact $(n-2)$-form ${ }^{13}$. In this expression, $\mathbf{Q}_{\xi}[\Phi]=-I_{\xi}^{n-1} \theta\left[\Phi ; \mathscr{L}_{\xi} \Phi\right]$ is called the NoetherWald surface charge.

Example For general relativity theory, the presymplectic potential (4.70) is given by

$$
\theta[g ; h]=\frac{\sqrt{-g}}{16 \pi G}\left(\nabla_{v} h^{\mu \nu}-\nabla^{\mu} h\right)\left(\mathrm{d}^{n-1} x\right)_{\mu},
$$

\footnotetext{
${ }^{13}$ In the definition (4.73), we assumed that the variational operator $\delta$ in front of the Noether-Wald charge does not see the possible field-dependence of the asymptotic Killing vectors $\xi^{\mu}$. Strictly speaking, one should write $\mathbf{k}_{\xi}^{I W}[\Phi ; \delta \Phi]=$ $-\delta \mathbf{Q}_{\xi}[\Phi]+\mathbf{Q}_{\delta \xi}[\Phi]-\iota_{\xi} \theta[\Phi ; \delta \Phi]$.
} 
where $h_{\mu \nu}=\delta g_{\mu \nu}$. Indices are lowered and raised by $g_{\mu \nu}$ and its inverse, and $h=h^{\mu}{ }_{\mu}$. From this expression, the Noether-Wald charge can be computed; we obtain

$$
\mathbf{Q}_{\xi}[g]=-I_{\xi}^{n-1} \theta\left[g ; \mathscr{L}_{\xi} g\right]=\frac{\sqrt{-g}}{8 \pi G} \nabla^{\mu} \xi^{v}\left(\mathrm{~d}^{n-2} x\right)_{\mu v}
$$

and we recognize the Komar charge. Finally, inserting these expression into (4.73) yields

$$
\mathbf{k}_{\xi}^{I W}[g ; h]=\frac{\sqrt{-g}}{8 \pi G}\left(\xi^{\mu} \nabla_{\sigma} h^{v \sigma}-\xi^{\mu} \nabla^{v} h+\xi_{\sigma} \nabla^{v} h^{\mu \sigma}+\frac{1}{2} h \nabla^{v} \xi^{\mu}-h^{\rho v} \nabla_{\rho} \xi^{\mu}\right)\left(\mathrm{d}^{n-2} x\right)_{\mu v} .
$$

Theorem [Conservation law] We have the following conservation law:

$$
\mathrm{dk}_{\xi}^{I W}[\Phi ; \delta \Phi] \approx \omega\left[\Phi ; \mathscr{L}_{\xi} \Phi, \delta \Phi\right],
$$

where, in the equality $\approx$, it is implied that $\Phi$ is a solution of the Euler-Lagrange equations and $\delta \Phi$ is a solution of the linearized Euler-Lagrange equations. Furthermore, $\omega\left[\Phi ; \mathscr{L}_{\xi} \Phi, \delta \Phi\right]=$ $i_{\mathscr{L}_{\xi} \Phi} \omega[\Phi ; \delta \Phi, \delta \Phi]=-\omega\left[\Phi ; \delta \Phi, \mathscr{L}_{\xi} \Phi\right]$.

This can be proved using Noether's second theorem (4.28) (see e.g. [63] for a detailed proof).

Remark In the covariant phase space formalism, the relation between non-integrability and nonconservation mentioned in the previous subsection is clear. Indeed,

$$
\begin{aligned}
\delta \not H_{\xi}[\Phi] & =\int_{\partial \Sigma} \delta \mathbf{k}_{\xi}^{I W}[\Phi, \delta \Phi] \\
& =\int_{\partial \Sigma} \delta \imath_{\xi} \theta[g, \delta g] \\
& =\int_{\partial \Sigma}-\imath_{\xi} \delta \theta[g, \delta g] \\
& =\int_{\partial \Sigma}-\imath_{\xi} \omega[g ; \delta g, \delta g],
\end{aligned}
$$

where we used (4.73) and (4.72) in the second and the fourth equality, respectively. The surface charge $\phi H_{\xi}[\Phi]$ is integrable only if $\delta \phi H_{\xi}[\Phi]=0$, if and only if

$$
\int_{\partial \Sigma} l_{\xi} \omega[g ; \delta g, \delta g]=0
$$

Therefore, from

$$
\mathrm{d} \phi H_{\xi}[\Phi]=\int_{\partial \Sigma} \mathrm{d} \mathbf{k}_{\xi}^{I W}[g, \delta g] \approx \int_{\partial \Sigma} \omega\left[\Phi ; \mathscr{L}_{\xi} \Phi, \delta \Phi\right],
$$

the non-conservation is controlled by $\omega[g, \delta g, \delta g]$ and is an obstruction for the integrability.

Remark As in the Barnich-Brandt procedure, the Iyer-Wald $(n-2)$-form (4.73) is defined up to an exact $(n-2)$-form. However, there is another source of ambiguity here coming from the definition of the presymplectic potential (4.70). In fact, we have the freedom to shift $\theta$ by an exact $(n-1)$-form as

$$
\theta[\Phi ; \delta \Phi] \rightarrow \theta[\Phi ; \delta \Phi]-\mathrm{d} \mathbf{Y}[\Phi ; \delta \Phi]
$$

where $\mathbf{Y}[\Phi ; \delta \Phi]$ is a $(n-2)$-form. This implies that the presymplectic form (4.72) is modified as

$$
\omega[\Phi ; \delta \Phi, \delta \Phi] \rightarrow \omega[\Phi ; \delta \Phi, \delta \Phi]+\mathrm{d} \delta \mathbf{Y}[\Phi ; \delta \Phi]
$$


where we used the fact that both $\mathrm{d}$ and $\delta$ are Grassmann odd. The Noether-Wald charge becomes

$$
\mathbf{Q}_{\xi}[\Phi] \rightarrow \mathbf{Q}_{\xi}[\Phi]+\mathbf{Y}\left[\Phi ; \mathscr{L}_{\xi} \Phi\right]
$$

up to an exact $(n-2)$-form which can be reabsorbed in the $(n-2)$-form ambiguity for $\mathbf{k}_{\xi}^{I W}$ discussed above. Therefore, this ambiguity modifies $\mathbf{k}_{F}^{I W}$ given in (4.73) by

$$
\mathbf{k}_{\xi}^{I W}[\Phi ; \delta \Phi] \rightarrow \mathbf{k}_{\xi}^{I W}[\Phi ; \delta \Phi]-\delta \mathbf{Y}\left[\Phi ; \mathscr{L}_{\xi} \Phi\right]-\imath_{\xi} \mathrm{d} \mathbf{Y}[\Phi ; \delta \Phi]
$$

Definition Let us introduce an important $(n-2)$-form which is involved in the relation between the Barnich-Brandt and Iyer-Wald prescriptions discussed in the remark below. We define

$$
\mathbf{E}[\Phi ; \delta \Phi, \delta \Phi]=-\frac{1}{2} I_{\delta \Phi}^{n-1} \theta=-\frac{1}{2} I_{\delta \Phi}^{n-1} I_{\delta \Phi}^{n} \mathbf{L} .
$$

Remark We now relate the Barnich-Brandt and the Iyer-Wald prescriptions to construct the $(n-$ 2)-form. Let us start from the expression (4.71) of the variation of the Lagrangian. We apply the homotopy operator on each side of the equality. We have

$$
\begin{aligned}
I_{\delta \Phi}^{n} \delta \mathbf{L} & =I_{\delta \Phi}^{n}\left(\delta \Phi \frac{\delta \mathbf{L}}{\delta \Phi}\right)-I_{\delta \Phi}^{n} \mathrm{~d} \theta \\
& =I_{\delta \Phi}^{n}\left(\delta \Phi \frac{\delta \mathbf{L}}{\delta \Phi}\right)-\delta \theta-\mathrm{d} I_{\delta \Phi}^{n-1} \theta
\end{aligned}
$$

Therefore,

$$
I_{\delta \Phi}^{n} \delta \mathbf{L}+\delta \theta=I_{\delta \Phi}^{n}\left(\delta \Phi \frac{\delta \mathbf{L}}{\delta \Phi}\right)-\mathrm{d} I_{\delta \Phi}^{n-1} \theta
$$

Since $\left[\delta, I_{\delta \Phi}^{n}\right]=0$ because $\delta^{2}=0$, the left-hand side of the last equality can be rewritten as $\delta I_{\delta \Phi}^{n} \mathbf{L}+$ $\delta \theta=2 \delta \theta=2 \omega$ where we used (4.70). Now, using (4.48) and (4.85), we obtain the relation between the presymplectic form $\omega$ and the invariant presymplectic current $\mathbf{W}$ as

$$
\omega[\Phi ; \delta \Phi, \delta \Phi]=\mathbf{W}[\Phi ; \delta \Phi, \delta \Phi]+\mathrm{d} \mathbf{E}[\Phi ; \delta \Phi, \delta \Phi]
$$

Contracting this relation with $i_{\mathscr{L}_{\xi} \Phi}$ results in

$$
\omega\left[\Phi ; \mathscr{L}_{\xi} \Phi, \delta \Phi\right]=\mathbf{W}\left[\Phi ; \mathscr{L}_{\xi} \Phi, \delta \Phi\right]+\mathrm{d} \mathbf{E}\left[\Phi ; \delta \Phi, \mathscr{L}_{\xi} \Phi\right]
$$

Finally, using the on-shell conservation laws (4.49) and (4.77), we obtain

$$
\mathbf{k}_{\xi}^{I W}[\Phi ; \delta \Phi] \approx \mathbf{k}_{\xi}[\Phi ; \delta \Phi]+\mathbf{E}\left[\Phi ; \delta \Phi, \mathscr{L}_{\xi} \Phi\right]
$$

up to an exact $(n-2)$-form. Therefore, the Barnich-Brandt $(n-2)$-form $\mathbf{k}_{\xi}[\Phi ; \delta \Phi]$ differs from the Iyer-Wald $(n-2)$-form $\mathbf{k}_{\xi}^{I W}[\Phi ; \delta \Phi]$ by the term $\mathbf{E}\left[\Phi ; \delta \Phi, \mathscr{L}_{\xi} \Phi\right]$. 
Examples We illustrate these concepts with the case of general relativity. The $(n-2)$-form $\mathbf{E}[\Phi ; \delta \Phi, \delta \Phi]$ can be computed using (4.85). We obtain

$$
\mathbf{E}[g ; \delta g, \delta g]=\frac{\sqrt{-g}}{32 \pi G}(\delta g)^{\mu}{ }_{\sigma} \wedge(\delta g)^{\sigma v}\left(\mathrm{~d}^{n-2} x\right)_{\mu \nu} .
$$

When contracted with $i_{\mathscr{L}_{\xi} g}$, this leads to

$$
\mathbf{E}\left[g ; \delta g, \mathscr{L}_{\xi} g\right]=-\frac{\sqrt{-g}}{16 \pi G}\left(\nabla^{\mu} \xi_{\sigma}+\nabla_{\sigma} \xi^{\mu}\right)(\delta g)^{\sigma v}\left(\mathrm{~d}^{n-2} x\right)_{\mu \nu},
$$

up to an exact $(n-2)$-form. This expression can also be obtained from (4.90) by comparing the explicit expressions (4.47) and (4.76). Notice that the difference between the Barnich-Brandt and the Iyer-Wald definitions (4.92) vanishes for a Killing vectors $\xi^{\mu}$. Furthermore, a simple computation shows that the $(n-2)$-form (4.91) vanishes in both the Fefferman-Graham gauge (3.8) and the Bondi gauge (3.10). Therefore, the Barnich-Brandt and the Iyer-Wald prescriptions lead to the same surface charges in these gauges. For an example where the two prescriptions do not coincide, see for instance, [12].

\section{Applications}

Asymptotic symmetries have a wide range of applications in theoretical physics. We briefly mention two of them and explain why the formalism presented above is relevant in these contexts.

\subsection{Holography}

The holographic principle states that quantum gravity can be described in terms of lowerdimensional dual quantum field theories [165, 167]. A concrete realization of the holographic principle asserts that the type IIB string theory living in the bulk spacetime $\operatorname{AdS}_{5} \times S^{5}$ is dual to the $\mathscr{N}=4$ supersymmetric Yang-Mills theory living on the four-dimensional spacetime boundary [126]. The gravitational theory is effectively living in the five-dimensional spacetime $\mathrm{AdS}_{5}$, the five dimensions of the factor $S^{5}$ being compactified. A first extension of this original holographic duality is the AdS/CFT correspondence which tells us that the gravitational theory living in the $(d+1)$-dimensional asymptotically AdS spacetime (AAdS2) is dual to a CFT living on the $d$ dimensional boundary. Other holographic dualities with different types of asymptotics have also been studied. A holographic dictionary enables one to interpret properties of the bulk theory in terms of the dual boundary theory. For example, the dictionary imposes the following relation between the symmetries of the two theories:

$$
\left[\begin{array}{c}
\text { Gauge symmetries in the bulk theory } \\
\Longleftrightarrow \\
\text { Global symmetries in the boundary theory. }
\end{array}\right]
$$

More specifically for us, consider a given bulk solution space with asymptotic symmetries. The correspondence tells us that a set of quantum field theories exist that are associated with the bulk solutions, such that in the UV regime, the global symmetries of these theories are exactly the 
asymptotic symmetries of the bulk solution space. Even if the AdS/CFT correspondence has not been proven yet, it has been verified in a number of situations and extended in various directions.

We now mention a famous hint in favor of this correspondence using the relation (5.1). Brown and Henneaux showed that the asymptotic symmetry group for asymptotically $\mathrm{AdS}_{3}$ spacetime with Dirichlet boundary conditions is given by the infinite-dimensional group of conformal transformations in two dimensions. Furthermore, they revealed that the associated surface charges are finite, are integrable, and exhibit a non-trivial central extension in their algebra. This Brown-Henneaux central charge is given by

$$
c=\frac{3 \ell}{2 G}
$$

where $\ell$ is the $\mathrm{AdS}_{3}$ radius. The AdS/CFT correspondence tells us that there is a set of twodimensional dual conformal field theories. The remarkable fact is that, when inserting the central charge (5.2) into the Cardy entropy formula valid for $2 d$ CFT [159], this reproduces exactly the entropy of three-dimensional BTZ black hole solutions [16,17].

The holographic principle is believed to hold in all types of asymptotics. In particular, in asymptotically flat spacetimes, from the correspondence (5.1), the dual theory would have BMS as the global symmetry. Important steps have been taken in this direction in three and four dimensions (see e.g. $[13,14,20,40,73,86,87,152]$ and references therein). Furthermore, in four-dimensional asymptotically flat spacetimes, traces of two-dimensional CFT seem to appear, enabling the use of well-known techniques of the AdS/CFT correspondence [15, 36, 57, 74, 82, 121, 132, 141, 150]. Notice that global BMS symmetry can be seen as a conformal Carroll symmetry $[84,85,91]$, which is especially relevant in the context of the fluid/gravity correspondence $[50,54,59,60,100,116]$.

\subsection{Infrared physics}

A connection has recently been established between various areas of gauge theories that are $a$ priori unrelated, namely asymptotic symmetries, soft theorems and memory effects (see [162] for a review). These three fields of research are often referred to as the three corners of the infrared triangle of gauge theories (see figure 2).

The first corner is the area of asymptotic symmetries that has been partially studied in these notes. The second corner is the topic of soft theorems $[96,122,173,174,178]$. These theorems state that any $(n+1)$-particles scattering amplitude involving a massless soft particle, namely a particle with momentum $q \rightarrow 0$ (that may be a photon, a gluon or a graviton), is equal to the $n$-particles scattering amplitude without the soft particle, multiplied by the soft factor, plus corrections of order $q^{0}$. We have

$$
\mathscr{M}_{n+1}\left(q, p_{1}, \ldots p_{n}\right)=S^{(0)} \mathscr{M}_{n}\left(p_{1}, \ldots p_{n}\right)+\mathscr{O}\left(q^{0}\right),
$$

where $S^{(0)} \sim q^{-1}$ is the soft factor whose precise form depends on the nature of the soft particle involved. Taking as soft particle a photon, gluon or graviton will respectively lead to the soft photon theorem, soft gluon theorem and soft graviton theorem. A remarkable property is that the soft factor is independent of the spin of the $n$ particles involved in the process. Furthermore, some so-called subleading soft theorems have been established for the different types of soft particles and they provide some information about the subleading terms in $q[48,98,118,123,175]$. They take the form

$$
\mathscr{M}_{n+1}\left(q, p_{1}, \ldots p_{n}\right)=\left(S^{(0)}+S^{(1)}\right) \mathscr{M}_{n}\left(p_{1}, \ldots p_{n}\right)+\mathscr{O}(q),
$$




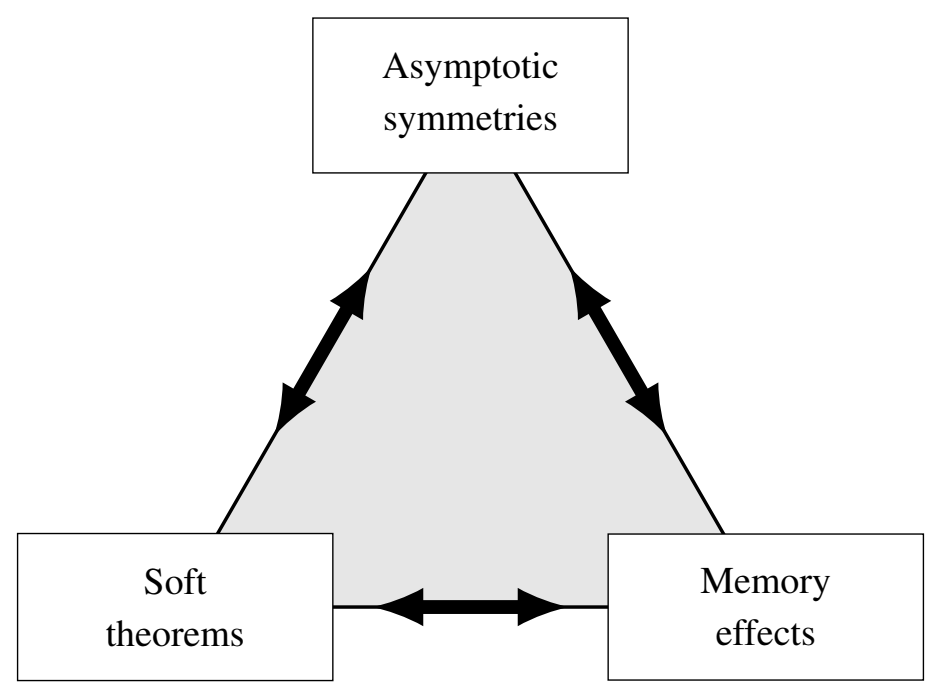

Figure 2: Infrared sector of gauge theories.

where $S^{(1)} \sim q^{0}$ is the subleading soft factor. Proposals for sub-subleading soft theorems can also be found $[49,76,180]$.

The third corner of the triangle is the topic of memory effects $[42,45,46,58,88,143,168$, $169,176,179]$. In gravity, the displacement memory effect occurs, for example, in the passage of gravitational waves. It can be shown that this produces a permanent shift in the relative positions of a pair of inertial detectors. This shift is controlled by a field in the metric that is turned on when the gravitational wave is passing through the spacetime region of interest. Considering the Bondi gauge in asymptotically flat spacetime with boundary conditions (AF3) (equations (3.15) with (3.18)), it can be shown that

$$
\Delta s^{A} \propto \Delta C_{A B}
$$

i.e. the angular displacement $\Delta s^{A}$ of two inertial observers in the asymptotic region is dictated by the field $C_{A B}$. Three processes can turn on the field $C_{A B}$ and trigger an observable displacement memory effect: a variation of the Bondi mass aspect $M$ (ordinary memory effect), a burst of gravitational waves controlled by the news $N_{A B}$ (Christodoulou effect), or a burst of null matter (null memory effect) [63]. The analogous memory effects can also be established in electrodynamics (electromagnetic memory effect) [140,166] and in Yang-Mills theory (color memory effect) [143] where a field is turned on as a result of a burst of energy passing through the region of interest, leading to an observable phenomenon. Notice that other memory effects have been identified in gravity $[65,80,92,129,136,142,147,148]$, including the spin memory effect and the refraction memory effect.

We now briefly discuss the relation between these different topics. It has been shown that if the quantum gravity $\mathscr{S}$-matrix is invariant under the BMS symmetry [161], then the Ward identity associated with the supertranslations is equivalent to the soft graviton theorem [108]. Furthermore, the displacement memory effect is equivalent to performing a supertranslation [163]. More precisely, the action of the supertranslation on the memory field $C_{A B}$ has the same effect as a burst of gravitational waves passing through the region of interest. This can be understood as a vac- 
uum transition process $[1,62,67,68,156]$. Finally, a Fourier transform enables us to relate the soft theorem with the memory effect, which closes the triangle. This triangle controlling the infrared structure of the theory has also been constructed for other gauge theories [125, 128, 143]. Furthermore, subleading infrared triangles have been uncovered and discussed [53, 65, 71, 115, 120, 125]. In particular, the Ward identities of superrotations have been shown to be equivalent to the subleading soft graviton theorem. Furthermore, the spin memory effect and the refraction memory effect have been related to the superrotations.

Finally, let us mention that this understanding of the infrared structure of quantum gravity is relevant to tackle the black hole information paradox [105]. Indeed, an infinite number of soft gravitons are produced in the process of black hole evaporation. Through the above correspondence, these soft gravitons are related with surface charges, called soft hairs, that have to be taken into account in the information storage [44,101,102,106,107,131].

\section{Acknowledgements}

I would like to thank Glenn Barnich, Luca Ciambelli, Geoffrey Compère, Adrien Fiorucci, Yannick Herfray, Kévin Nguyen and Céline Zwikel for their comments on the manuscript and discussions on related subjects. I would also like to thank the participants of the XV Modave Summer School 2019 for this stimulating edition. This work is supported by the FRIA (F.R.S.FNRS, Belgium).

\section{A. Diffeomorphism between Bondi and Fefferman-Graham gauges}

The diffeomorphism between Bondi and Fefferman-Graham gauges in asymptotically (A) $\mathrm{dS}_{4}$ spacetime has been worked out explicitly in [66,149]. In this appendix, we briefly recall how the solution space (3.30) associated with the preliminary boundary condition (3.19) in the FeffermanGraham gauge matches with the solution space (3.43) associated with the preliminary boundary condition (3.20) in the Bondi gauge through this diffeomorphism. The components of the threedimensional boundary metric $g_{a b}^{(0)}$ can be expressed in terms of the functions of the Bondi gauge as

$$
g_{t t}^{(0)}=\frac{\Lambda}{3} e^{4 \beta_{0}}+U_{0}^{C} U_{C}^{0}, \quad g_{t A}^{(0)}=-U_{A}^{0}, \quad g_{A B}^{(0)}=q_{A B} .
$$

Furthermore, the degrees of freedom of the stress-energy tensor $T_{a b}$ are related to degrees of freedom of the Bondi gauge as

$$
T_{t t} \sim M, \quad T_{t A} \sim N_{A}, \quad T_{A B} \sim \mathscr{E}_{A B} .
$$

The precise relations can be found in the references. Notice that the constraint $D_{(0)}^{a} T_{a b}=0$ translated in terms of the functions in the Bondi gauge gives the evolution constraints with respect to the $u$ coordinate for the Bondi mass aspect $M$ and the angular momentum aspect $N_{A}$.

\section{B. Useful results and conventions}

In this appendix, we establish some important frameworks and conventions. The aim of this formalism is to manipulate some local expressions, as this is convenient in field theory. We closely follow [61,63]. 


\section{B.1 Jet bundles}

Let $M$ be the $n$-dimensional spacetime with local coordinates $x^{\mu}(\mu=0, \ldots, n-1)$. The fields, written as $\Phi=\left(\phi^{i}\right)$, are supposed to be Grassmann even. The jet space $J$ consists in the fields and the symmetrized derivatives of the fields $\left(\Phi, \Phi_{\mu}, \Phi_{\mu \nu}, \ldots\right)$, where $\Phi_{\mu_{1} \ldots \mu_{k}}=\frac{\partial}{\mu_{\mu_{1}}} \ldots \frac{\partial}{\partial_{\mu_{k}}} \Phi$. The symmetrized derivative is defined as

$$
\frac{\partial \tilde{\Phi}_{v_{1} \ldots v_{k}}}{\partial \Phi_{\mu_{1} \ldots \mu_{k}}}=\delta_{\left(\mu_{1}\right.}^{v_{1}} \ldots \delta_{\left.\mu_{k}\right)}^{v_{k}} \delta_{\Phi}^{\tilde{\Phi}}
$$

In the jet space, the cotangent space at a point is generated by the variations of the fields and their derivatives at that point, namely $\left(\delta \Phi, \delta \Phi_{\mu}, \delta \Phi_{\mu \nu}, \ldots\right)$. The variational operator is defined as

$$
\delta=\sum_{k \geq 0} \delta \Phi_{\mu_{1} \ldots \mu_{k}} \frac{\partial}{\partial \Phi_{\mu_{1} \ldots \mu_{k}}} .
$$

We choose all the $\delta \Phi, \delta \Phi_{\mu}, \delta \Phi_{\mu \nu}, \ldots$ to be Grassmann odd, which implies that $\delta^{2}=0$. Hence, $\delta$ is seen as an exterior derivative on the jet space.

Now, we define the jet bundle as the fiber bundle with local trivialization $\left(x^{\mu}, \Phi, \Phi_{\mu}, \Phi_{\mu \nu}, \ldots\right)$. Locally, the total space of the jet bundle looks like $M \times J$. A section of this fiber bundle is a map $x \rightarrow\left(\Phi(x), \Phi_{\mu}(x), \Phi_{\mu v}(x), \ldots\right)$. The horizontal derivative is defined as

$$
\mathrm{d}=\mathrm{d} x^{\mu} \partial_{\mu}, \quad \text { where } \quad \partial_{\mu}=\frac{\partial}{\partial x^{\mu}}+\sum_{k \geq 0} \Phi_{\mu v_{1} \ldots v_{k}} \frac{\partial}{\partial \Phi_{v_{1} \ldots v_{k}}} .
$$

In this perspective, the variational operator can also be seen as the vertical derivative, i.e. the derivative along the fibers. The exterior derivative on the total space can be defined as $\mathrm{d}_{T o t}=\mathrm{d}+\delta$. Notice that both $\mathrm{d}$ and $\delta$ are Grassmann odd and they anti-commute, namely

$$
\mathrm{d} \delta=-\delta \mathrm{d} .
$$

On the jet bundle, we write $\Omega^{p, q}$ the set of functions that are $p$-forms with respect to the spacetime and $q$-forms with respect to the jet space ${ }^{14}$.

\section{B.2 Some operators}

In this subsection, we introduce some additional operators used in the text and discuss their properties.

The Euler-Lagrange derivative of a local function $f$, i.e. a function on the total space of the jet bundle $f=f\left[x, \Phi, \Phi_{\mu}, \Phi_{\mu \nu}, \ldots\right]$, is defined as

$$
\frac{\delta f}{\delta \Phi}=\sum_{k \geq 0}(-1)^{k} \partial_{\mu_{1}} \ldots \partial_{\mu_{k}}\left(\frac{\partial f}{\partial \Phi_{\mu_{1} \ldots \mu_{k}}}\right) .
$$

This operator satisfies

$$
\frac{\delta f}{\delta \Phi}=0 \quad \Leftrightarrow \quad f=\partial_{\mu} j^{\mu},
$$

\footnotetext{
${ }^{14}$ One often refers to a variational bicomplex structure
} 
where $j^{\mu}$ is a local function (for a proof, see e.g. section 1.2 of [26]).

The variation under a transformation of characteristic $Q$ (i.e. $\delta_{Q} \Phi=Q$ ) is given by

$$
\delta_{Q} f=\sum_{k \geq 0}\left(\partial_{\mu_{1}} \ldots \partial_{\mu_{k}} Q\right) \frac{\partial}{\partial \Phi_{\mu_{1} \ldots \mu_{k}}}+\left(\partial_{\mu_{1}} \ldots \partial_{\mu_{k}} \delta Q\right) \frac{\partial}{\partial \delta \Phi_{\mu_{1} \ldots \mu_{k}}} .
$$

The Lie bracket of characteristics is defined by $\left[Q_{1}, Q_{2}\right]=\delta_{Q_{1}} Q_{2}-\delta_{Q_{2}} Q_{1}$ and satisfies $\left[\delta_{Q_{1}}, \delta_{Q_{2}}\right]=$ $\delta_{\left[Q_{1}, Q_{2}\right]}$. A contracted variation of this type is Grassmann even and we have

$$
\delta_{Q} \mathrm{~d}=\mathrm{d} \delta_{Q}, \quad \delta \delta_{Q}=\delta_{Q} \delta
$$

We also have the following relation between the variation under a transformation of characteristic $Q$ and the Euler-Lagrange derivative:

$$
\delta_{Q} \frac{\delta f}{\delta \Phi}=\frac{\delta}{\delta \Phi}\left(\delta_{Q} f\right)-\sum_{k \geq 0}(-1)^{k} \partial_{\mu_{1}} \ldots \partial_{\mu_{k}}\left(\frac{\partial Q}{\partial \Phi_{\mu_{1} \ldots \mu_{k}}} \frac{\delta f}{\delta \Phi}\right) .
$$

Let $\alpha$ be a $(n-k)$-form on the space-time $M$. We use the notation

$$
\alpha=\alpha^{\mu_{1} \ldots \mu_{k}}\left(\mathrm{~d}^{n-k} x\right)_{\mu_{1} \ldots \mu_{k}}
$$

where

$$
\left(\mathrm{d}^{n-k} x\right)_{\mu_{1} \ldots \mu_{k}}=\frac{1}{k !(n-k) !} \varepsilon_{\mu_{1} \ldots \mu_{k} v_{1} \ldots v_{n-k}} \mathrm{~d} x^{v_{1}} \wedge \ldots \wedge \mathrm{d} x^{v_{n-k}}
$$

and where $\varepsilon_{\mu_{1} \ldots \mu_{n}}$ is completely antisymmetric and $\varepsilon_{01 \ldots n-1}=1$. We can check that

$$
\mathrm{d} \alpha=\partial_{\sigma} \alpha^{\left[\mu_{1} \ldots \mu_{k-1} \sigma\right]}\left(\mathrm{d}^{n-k+1} x\right)_{\mu_{1} \ldots \mu_{k-1}} .
$$

The interior product of a spacetime form with respect to a vector field $\xi$ is defined as

$$
\iota_{\xi}=\xi^{\mu} \frac{\partial}{\partial \mathrm{d} x^{\mu}} .
$$

Notice that we can also define the interior product of a jet space form with respect to a characteristic $Q$ as

$$
i_{Q}=\sum_{k \geq 0}\left(\partial_{\mu_{1}} \ldots \partial_{\mu_{k}} Q\right) \frac{\partial}{\partial \delta \Phi_{\mu_{1} \ldots \mu_{k}}}
$$

It satisfies

$$
i_{Q} \delta+\delta i_{Q}=\delta_{Q}, \quad i_{Q_{1}} \delta_{Q_{2}}-\delta_{Q_{2}} i_{Q_{1}}=i_{\left[Q_{1}, Q_{2}\right]} .
$$

The homotopy operator $I_{\delta \Phi}^{p}: \Omega^{p, q} \mapsto \Omega^{p-1, q+1}$ is defined as

$$
I_{\delta \Phi}^{p} \alpha=\sum_{k \geq 0} \frac{k+1}{n-p+k+1} \partial_{\mu_{1}} \ldots \partial_{\mu_{k}}\left(\delta \Phi \frac{\delta}{\delta \Phi_{\mu_{1} \ldots \mu_{k} v}} \frac{\partial \alpha}{\partial \mathrm{d} x^{v}}\right)
$$

for $\alpha \in \Omega^{p, q}$. This operator satisfies the following relations

$$
\begin{aligned}
& \delta=\delta \Phi \frac{\delta}{\delta \Phi}-\mathrm{d} I_{\delta \Phi}^{n} \quad \text { when acting on spacetime } n \text {-forms, } \\
& \delta=I_{\delta \Phi}^{p+1} \mathrm{~d}-\mathrm{d} I_{\delta \Phi}^{p} \quad \text { when acting on spacetime } p \text {-forms }(p<n) .
\end{aligned}
$$


Furthermore,

$$
\delta I_{\delta \Phi}^{p}=I_{\delta \Phi}^{p} \delta
$$

Notice that the homotopy operator is used to prove the algebraic Poincare lemma (4.16).

Similarly, the homotopy operator with respect to gauge parameters $F$ is defined as $I_{F}^{p}: \Omega^{p, q} \mapsto$ $\Omega^{p-1, q}$, where

$$
I_{F}^{p} \alpha=\sum_{k \geq 0} \frac{k+1}{n-p+k+1} \partial_{\mu_{1}} \ldots \partial_{\mu_{k}}\left(F \frac{\delta}{\delta F_{\mu_{1} \ldots \mu_{k} v}} \frac{\partial \alpha}{\partial \mathrm{d} x^{v}}\right)
$$

It satisfies

$$
I_{F}^{p+1} \mathrm{~d}+\mathrm{d} I_{F}^{p}=1
$$

\section{References}

[1] Eugene Adjei, William Donnelly, Victor Py, and Antony J. Speranza. Cosmic footballs from superrotations. 2019.

[2] Hamid Afshar, Erfan Esmaeili, and M. M. Sheikh-Jabbari. Asymptotic Symmetries in $p$-Form Theories. JHEP, 05:042, 2018.

[3] Ofer Aharony, Steven S. Gubser, Juan Martin Maldacena, Hirosi Ooguri, and Yaron Oz. Large N field theories, string theory and gravity. Phys. Rept., 323:183-386, 2000.

[4] A. Ashtekar and R. O. Hansen. A unified treatment of null and spatial infinity in general relativity. I - Universal structure, asymptotic symmetries, and conserved quantities at spatial infinity. J. Math. Phys., 19:1542-1566, 1978.

[5] A. Ashtekar and Joseph D. Romano. Spatial infinity as a boundary of space-time. Class. Quant. Grav., 9:1069-1100, 1992.

[6] A. Ashtekar and M. Streubel. Symplectic Geometry of Radiative Modes and Conserved Quantities at Null Infinity. Proc. Roy. Soc. Lond., A376:585-607, 1981.

[7] Abhay Ashtekar. Geometry and Physics of Null Infinity. 2014.

[8] Abhay Ashtekar and Sina Bahrami. Asymptotics with a positive cosmological constant. IV. The no-incoming radiation condition. Phys. Rev., D100(2):024042, 2019.

[9] Abhay Ashtekar, Béatrice Bonga, and Aruna Kesavan. Asymptotics with a positive cosmological constant: I. Basic framework. Class. Quant. Grav., 32(2):025004, 2015.

[10] Abhay Ashtekar, Béatrice Bonga, and Aruna Kesavan. Asymptotics with a positive cosmological constant. II. Linear fields on de Sitter spacetime. Phys. Rev., D92(4):044011, 2015.

[11] Abhay Ashtekar, Béatrice Bonga, and Aruna Kesavan. Asymptotics with a positive cosmological constant: III. The quadrupole formula. Phys. Rev., D92(10):104032, 2015.

[12] Tatsuo Azeyanagi, Geoffrey Compere, Noriaki Ogawa, Yuji Tachikawa, and Seiji Terashima. Higher-Derivative Corrections to the Asymptotic Virasoro Symmetry of 4d Extremal Black Holes. Prog. Theor. Phys., 122:355-384, 2009.

[13] Arjun Bagchi. Correspondence between Asymptotically Flat Spacetimes and Nonrelativistic Conformal Field Theories. Phys. Rev. Lett., 105:171601, 2010.

[14] Arjun Bagchi, Rudranil Basu, Daniel Grumiller, and Max Riegler. Entanglement entropy in Galilean conformal field theories and flat holography. Phys. Rev. Lett., 114(11):111602, 2015. 
[15] Adam Ball, Elizabeth Himwich, Sruthi A. Narayanan, Sabrina Pasterski, and Andrew Strominger. Uplifting $\mathrm{AdS}_{3} / \mathrm{CFT}_{2}$ to flat space holography. JHEP, 08:168, 2019.

[16] Maximo Banados, Marc Henneaux, Claudio Teitelboim, and Jorge Zanelli. Geometry of the $(2+1)$ black hole. Phys. Rev., D48:1506-1525, 1993. [Erratum: Phys. Rev.D88,069902(2013)].

[17] Maximo Banados, Claudio Teitelboim, and Jorge Zanelli. The Black hole in three-dimensional space-time. Phys. Rev. Lett., 69:1849-1851, 1992.

[18] Glenn Barnich. Boundary charges in gauge theories: Using Stokes theorem in the bulk. Class. Quant. Grav., 20:3685-3698, 2003.

[19] Glenn Barnich. A Note on gauge systems from the point of view of Lie algebroids. AIP Conf. Proc., 1307(1):7-18, 2010.

[20] Glenn Barnich. Entropy of three-dimensional asymptotically flat cosmological solutions. JHEP, 10:095, 2012.

[21] Glenn Barnich. Centrally extended BMS4 Lie algebroid. JHEP, 06:007, 2017.

[22] Glenn Barnich and Friedemann Brandt. Covariant theory of asymptotic symmetries, conservation laws and central charges. Nucl. Phys., B633:3-82, 2002.

[23] Glenn Barnich, Friedemann Brandt, and Marc Henneaux. Local BRST cohomology in Einstein Yang-Mills theory. Nucl. Phys., B455:357-408, 1995.

[24] Glenn Barnich, Friedemann Brandt, and Marc Henneaux. Local BRST cohomology in the antifield formalism. 1. General theorems. Commun. Math. Phys., 174:57-92, 1995.

[25] Glenn Barnich and Geoffrey Compere. Surface charge algebra in gauge theories and thermodynamic integrability. J. Math. Phys., 49:042901, 2008.

[26] Glenn Barnich and Fabrizio Del Monte. Introduction to Classical Gauge Field Theory and to Batalin-Vilkovisky Quantization. 2018.

[27] Glenn Barnich, Andres Gomberoff, and Hernan A. Gonzalez. Three-dimensional Bondi-Metzner-Sachs invariant two-dimensional field theories as the flat limit of Liouville theory. Phys. Rev., D87(12):124032, 2013.

[28] Glenn Barnich, Hernan A. Gonzalez, and Patricio Salgado-Rebolledo. Geometric actions for three-dimensional gravity. Class. Quant. Grav., 35(1):014003, 2018.

[29] Glenn Barnich and Pierre-Henry Lambert. A note on the Newman-Unti group. Adv. Math. Phys., 2012:197385, 2012.

[30] Glenn Barnich and Pierre-Henry Lambert. Einstein-Yang-Mills theory: Asymptotic symmetries. Phys. Rev., D88:103006, 2013.

[31] Glenn Barnich, Pujian Mao, and Romain Ruzziconi. Conserved currents in the Cartan formulation of general relativity. In About Various Kinds of Interactions: Workshop in honour of Professor Philippe Spindel Mons, Belgium, June 4-5, 2015, 2016.

[32] Glenn Barnich, Pujian Mao, and Romain Ruzziconi. BMS Current Algebra in the Context of the Newman-Penrose Formalism. 2019.

[33] Glenn Barnich and Blagoje Oblak. Holographic positive energy theorems in three-dimensional gravity. Class. Quant. Grav., 31:152001, 2014. 
[34] Glenn Barnich and Blagoje Oblak. Notes on the BMS group in three dimensions: II. Coadjoint representation. JHEP, 03:033, 2015.

[35] Glenn Barnich and Cédric Troessaert. Finite BMS transformations. JHEP, 03:167, 2016.

[36] Glenn Barnich and Cedric Troessaert. Aspects of the BMS/CFT correspondence. JHEP, 05:062, 2010.

[37] Glenn Barnich and Cédric Troessaert. Supertranslations call for superrotations. PoS, CNCFG2010:010, 2010. [Ann. U. Craiova Phys.21,S11(2011)].

[38] Glenn Barnich and Cédric Troessaert. Symmetries of asymptotically flat 4 dimensional spacetimes at null infinity revisited. Phys. Rev. Lett., 105:111103, 2010.

[39] Glenn Barnich and Cedric Troessaert. BMS charge algebra. JHEP, 12:105, 2011.

[40] Rudranil Basu, Stéphane Detournay, and Max Riegler. Spectral Flow in 3D Flat Spacetimes. JHEP, 12:134, 2017.

[41] Luc Blanchet. Gravitational Radiation from Post-Newtonian Sources and Inspiralling Compact Binaries. Living Rev. Rel., 17:2, 2014.

[42] Luc Blanchet and Thibault Damour. Hereditary effects in gravitational radiation. Phys. Rev., D46:4304-4319, 1992.

[43] H. Bondi, M. G. J. van der Burg, and A. W. K. Metzner. Gravitational waves in general relativity. 7. Waves from axisymmetric isolated systems. Proc. Roy. Soc. Lond., A269:21-52, 1962.

[44] Raphael Bousso and Massimo Porrati. Soft Hair as a Soft Wig. Class. Quant. Grav., 34(20):204001, 2017.

[45] V. B. Braginsky and L. P. Grishchuk. Kinematic Resonance and Memory Effect in Free Mass Gravitational Antennas. Sov. Phys. JETP, 62:427-430, 1985. [Zh. Eksp. Teor. Fiz.89,744(1985)].

[46] Vladimir B. Braginsky and Kip S. Thorne. Gravitational-wave bursts with memory and experimental prospects. Nature, 327:123-125, 1987.

[47] J. David Brown and M. Henneaux. Central Charges in the Canonical Realization of Asymptotic Symmetries: An Example from Three-Dimensional Gravity. Commun. Math. Phys., 104:207-226, 1986.

[48] T. H. Burnett and Norman M. Kroll. Extension of the low soft photon theorem. Phys. Rev. Lett., 20:86, 1968.

[49] Freddy Cachazo and Andrew Strominger. Evidence for a New Soft Graviton Theorem. 2014.

[50] Marco M. Caldarelli, Robert G. Leigh, Anastasios C. Petkou, P. Marios Petropoulos, Valentina Pozzoli, and Konstadinos Siampos. Vorticity in holographic fluids. PoS, CORFU2011:076, 2011.

[51] Miguel Campiglia and Alok Laddha. Asymptotic symmetries and subleading soft graviton theorem. Phys. Rev., D90(12):124028, 2014.

[52] Miguel Campiglia and Alok Laddha. New symmetries for the Gravitational S-matrix. JHEP, 04:076, 2015.

[53] Miguel Campiglia and Alok Laddha. Subleading soft photons and large gauge transformations. JHEP, 11:012, 2016.

[54] Andrea Campoleoni, Luca Ciambelli, Charles Marteau, P. Marios Petropoulos, and Konstantinos Siampos. Two-dimensional fluids and their holographic duals. Nucl. Phys., B946:114692, 2019. 
[55] Federico Capone and Marika Taylor. Cosmic branes and asymptotic structure. 2019.

[56] Venkatesa Chandrasekaran, Éanna E. Flanagan, and Kartik Prabhu. Symmetries and charges of general relativity at null boundaries. JHEP, 11:125, 2018.

[57] Clifford Cheung, Anton de la Fuente, and Raman Sundrum. 4D scattering amplitudes and asymptotic symmetries from 2D CFT. JHEP, 01:112, 2017.

[58] D. Christodoulou. Nonlinear nature of gravitation and gravitational wave experiments. Phys. Rev. Lett., 67:1486-1489, 1991.

[59] Luca Ciambelli, Robert G. Leigh, Charles Marteau, and P. Marios Petropoulos. Carroll Structures, Null Geometry and Conformal Isometries. Phys. Rev., D100(4):046010, 2019.

[60] Luca Ciambelli, Anastasios C. Petkou, P. Marios Petropoulos, and Konstantinos Siampos. The Robinson-Trautman spacetime and its holographic fluid. PoS, CORFU2016:076, 2017.

[61] Geoffrey Compere. Symmetries and conservation laws in Lagrangian gauge theories with applications to the mechanics of black holes and to gravity in three dimensions. $\mathrm{PhD}$ thesis, Brussels U., 2007.

[62] Geoffrey Compère. Bulk supertranslation memories: a concept reshaping the vacua and black holes of general relativity. Int. J. Mod. Phys., D25(12):1644006, 2016.

[63] Geoffrey Compère. Advanced Lectures on General Relativity. Lect. Notes Phys., 952:150, 2019.

[64] Geoffrey Compère. Infinite towers of supertranslation and superrotation memories. Phys. Rev. Lett., 123(2):021101, 2019.

[65] Geoffrey Compère, Adrien Fiorucci, and Romain Ruzziconi. Superboost transitions, refraction memory and super-Lorentz charge algebra. JHEP, 11:200, 2018.

[66] Geoffrey Compère, Adrien Fiorucci, and Romain Ruzziconi. The $\Lambda$-BMS 4 group of $\mathrm{dS}_{4}$ and new boundary conditions for $\mathrm{AdS}_{4} .2019$.

[67] Geoffrey Compère and Jiang Long. Classical static final state of collapse with supertranslation memory. Class. Quant. Grav., 33(19):195001, 2016.

[68] Geoffrey Compère and Jiang Long. Vacua of the gravitational field. JHEP, 07:137, 2016.

[69] Geoffrey Compère and Donald Marolf. Setting the boundary free in AdS/CFT. Class. Quant. Grav., 25:195014, 2008.

[70] Geoffrey Compère, R. Oliveri, and A. Seraj. Gravitational multipole moments from Noether charges. JHEP, 05:054, 2018.

[71] Eduardo Conde and Pujian Mao. Remarks on asymptotic symmetries and the subleading soft photon theorem. Phys. Rev., D95(2):021701, 2017.

[72] Marius Crainic and Rui Loja Fernandes. Lectures on Integrability of Lie Brackets. 2006.

[73] Claudio Dappiaggi. BMS field theory and holography in asymptotically flat space-times. JHEP, 11:011, 2004.

[74] Jan de Boer and Sergey N. Solodukhin. A Holographic reduction of Minkowski space-time. Nucl. Phys., B665:545-593, 2003.

[75] Stéphane Detournay, P. Marios Petropoulos, and Céline Zwikel. Asymptotic Symmetries of Three-Dimensional Black Strings. JHEP, 06:131, 2019. 
[76] Paolo Di Vecchia, Raffaele Marotta, and Matin Mojaza. Subsubleading soft theorems of gravitons and dilatons in the bosonic string. JHEP, 06:054, 2016.

[77] Laura Donnay and Gaston Giribet. Cosmological horizons, Noether charges and entropy. Class. Quant. Grav., 36(16):165005, 2019.

[78] Laura Donnay, Gaston Giribet, Hernán A. González, and Miguel Pino. Extended Symmetries at the Black Hole Horizon. JHEP, 09:100, 2016.

[79] Laura Donnay, Gaston Giribet, Hernan A. Gonzalez, and Miguel Pino. Supertranslations and Superrotations at the Black Hole Horizon. Phys. Rev. Lett., 116(9):091101, 2016.

[80] Laura Donnay, Gaston Giribet, Hernán A. González, and Andrea Puhm. Black hole memory effect. Phys. Rev., D98(12):124016, 2018.

[81] Laura Donnay and Charles Marteau. Carrollian Physics at the Black Hole Horizon. Class. Quant. Grav., 36(16):165002, 2019.

[82] Laura Donnay, Andrea Puhm, and Andrew Strominger. Conformally Soft Photons and Gravitons. JHEP, 01:184, 2019.

[83] T. Dray and M. Streubel. Angular momentum at null infinity. Class. Quant. Grav., 1(1):15-26, 1984.

[84] C. Duval, G. W. Gibbons, and P. A. Horvathy. Conformal Carroll groups. J. Phys., A47(33):335204, 2014.

[85] C. Duval, G. W. Gibbons, and P. A. Horvathy. Conformal Carroll groups and BMS symmetry. Class. Quant. Grav., 31:092001, 2014.

[86] Reza Fareghbal and Ali Naseh. Flat-Space Energy-Momentum Tensor from BMS/GCA Correspondence. JHEP, 03:005, 2014.

[87] Reza Fareghbal and Ali Naseh. Aspects of Flat/CCFT Correspondence. Class. Quant. Grav., 32:135013, 2015.

[88] Marc Favata. The gravitational-wave memory effect. Class. Quant. Grav., 27:084036, 2010.

[89] Charles Fefferman and C. Robin Graham. Conformal invariants. In Élie Cartan et les mathématiques d'aujourd'hui - Lyon, 25-29 juin 1984, number S131 in Astérisque, pages 95-116. Société mathématique de France, 1985.

[90] Charles Fefferman and C. Robin Graham. The ambient metric. arXiv e-prints, page arXiv:0710.0919, Oct 2007.

[91] José Figueroa-O’Farrill, Ross Grassie, and Stefan Prohazka. Geometry and BMS Lie algebras of spatially isotropic homogeneous spacetimes. JHEP, 08:119, 2019.

[92] Eanna E. Flanagan, Alexander M. Grant, Abraham I. Harte, and David A. Nichols. Persistent gravitational wave observables: general framework. Phys. Rev., D99(8):084044, 2019.

[93] Éanna É. Flanagan and David A. Nichols. Conserved charges of the extended Bondi-Metzner-Sachs algebra. Phys. Rev., D95(4):044002, 2017.

[94] Éanna É. Flanagan, Kartik Prabhu, and Ibrahim Shehzad. Extensions of the asymptotic symmetry algebra of general relativity. 2019.

[95] Ernesto Frodden and Diego Hidalgo. Surface Charges Toolkit for Gravity. 2019.

[96] Murray Gell-Mann and M. L. Goldberger. Scattering of low-energy photons by particles of spin 1/2. Phys. Rev., 96:1433-1438, 1954. 
[97] Hadi Godazgar, Mahdi Godazgar, and C. N. Pope. Subleading BMS charges and fake news near null infinity. JHEP, 01:143, 2019.

[98] David J. Gross and Roman Jackiw. Low-Energy Theorem for Graviton Scattering. Phys. Rev., 166:1287-1292, 1968.

[99] Daniel Grumiller, Alfredo Pérez, M. M. Sheikh-Jabbari, Ricardo Troncoso, and Céline Zwikel. Spacetime structure near generic horizons and soft hair. 2019.

[100] Michael Haack and Amos Yarom. Nonlinear viscous hydrodynamics in various dimensions using AdS/CFT. JHEP, 10:063, 2008.

[101] Sasha Haco, Stephen W. Hawking, Malcolm J. Perry, and Andrew Strominger. Black Hole Entropy and Soft Hair. JHEP, 12:098, 2018.

[102] Sasha Haco, Malcolm J. Perry, and Andrew Strominger. Kerr-Newman Black Hole Entropy and Soft Hair. 2019.

[103] R. O. Hansen, E. T. Newman, R. Penrose, and K. P. Tod. The Metric and Curvature Properties of H Space. Proc. Roy. Soc. Lond., A363:445-468, 1978.

[104] Daniel Harlow and Jie-Qiang Wu. Covariant phase space with boundaries. 2019.

[105] S. W. Hawking. Breakdown of Predictability in Gravitational Collapse. Phys. Rev., D14:2460-2473, 1976.

[106] Stephen W. Hawking, Malcolm J. Perry, and Andrew Strominger. Soft Hair on Black Holes. Phys. Rev. Lett., 116(23):231301, 2016.

[107] Stephen W. Hawking, Malcolm J. Perry, and Andrew Strominger. Superrotation Charge and Supertranslation Hair on Black Holes. JHEP, 05:161, 2017.

[108] Temple He, Vyacheslav Lysov, Prahar Mitra, and Andrew Strominger. BMS supertranslations and Weinbergś soft graviton theorem. JHEP, 05:151, 2015.

[109] Temple He, Prahar Mitra, Achilleas P. Porfyriadis, and Andrew Strominger. New Symmetries of Massless QED. JHEP, 10:112, 2014.

[110] M. Henneaux and C. Teitelboim. Asymptotically anti-De Sitter Spaces. Commun. Math. Phys., 98:391-424, 1985

[111] Marc Henneaux and Cédric Troessaert. Asymptotic symmetries of electromagnetism at spatial infinity. JHEP, 05:137, 2018.

[112] Marc Henneaux and Cédric Troessaert. BMS Group at Spatial Infinity: the Hamiltonian (ADM) approach. JHEP, 03:147, 2018.

[113] Marc Henneaux and Cédric Troessaert. The asymptotic structure of gravity at spatial infinity in four spacetime dimensions. 2019.

[114] Yannick Herfray. Asymptotic Shear and the Intrinsic Geometry of Null-Infinity. 2020.

[115] Elizabeth Himwich, Zahra Mirzaiyan, and Sabrina Pasterski. A Note on the Subleading Soft Graviton. 2019.

[116] Veronika E. Hubeny, Shiraz Minwalla, and Mukund Rangamani. The fluid/gravity correspondence. In Black holes in higher dimensions, pages 348-383, 2012. [,817(2011)].

[117] Vivek Iyer and Robert M. Wald. Some properties of Noether charge and a proposal for dynamical black hole entropy. Phys. Rev., D50:846-864, 1994. 
[118] R. Jackiw. Low-Energy Theorems for Massless Bosons: Photons and Gravitons. Phys. Rev., 168:1623-1633, 1968.

[119] John David Jackson. Classical Electrodynamics. Wiley, 1998.

[120] Daniel Kapec, Vyacheslav Lysov, Sabrina Pasterski, and Andrew Strominger. Semiclassical Virasoro symmetry of the quantum gravity $\mathscr{S}$-matrix. JHEP, 08:058, 2014.

[121] Daniel Kapec, Prahar Mitra, Ana-Maria Raclariu, and Andrew Strominger. 2D Stress Tensor for 4D Gravity. Phys. Rev. Lett., 119(12):121601, 2017.

[122] F. E. Low. Scattering of light of very low frequency by systems of spin 1/2. Phys. Rev., 96:1428-1432, 1954.

[123] F. E. Low. Bremsstrahlung of very low-energy quanta in elementary particle collisions. Phys. Rev., 110:974-977, 1958.

[124] H. Lu, Pujian Mao, and Jun-Bao Wu. Asymptotic Structure of Einstein-Maxwell-Dilaton Theory and Its Five Dimensional Origin. 2019.

[125] Vyacheslav Lysov, Sabrina Pasterski, and Andrew Strominger. Lowś Subleading Soft Theorem as a Symmetry of QED. Phys. Rev. Lett., 113(11):111601, 2014.

[126] Juan Martin Maldacena. The Large N limit of superconformal field theories and supergravity. Int. J. Theor. Phys., 38:1113-1133, 1999. [Adv. Theor. Math. Phys.2,231(1998)].

[127] Pujian Mao. Asymptotics with a cosmological constant: The solution space. Phys. Rev., D99(10):104024, 2019.

[128] Pujian Mao and Jun-Bao Wu. Note on asymptotic symmetries and soft gluon theorems. Phys. Rev., D96(6):065023, 2017.

[129] Pujian Mao and Xiaoning Wu. More on gravitational memory. JHEP, 05:058, 2019.

[130] Donald Marolf, William Kelly, and Sebastian Fischetti. Conserved Charges in Asymptotically (Locally) AdS Spacetimes. In Abhay Ashtekar and Vesselin Petkov, editors, Springer Handbook of Spacetime, pages 381-407. 2014.

[131] Mehrdad Mirbabayi and Massimo Porrati. Dressed Hard States and Black Hole Soft Hair. Phys. Rev. Lett., 117(21):211301, 2016.

[132] Rashmish K. Mishra and Raman Sundrum. Asymptotic Symmetries, Holography and Topological Hair. JHEP, 01:014, 2018.

[133] E. T. Newman and R. Penrose. New conservation laws for zero rest-mass fields in asymptotically flat space-time. Proc. Roy. Soc. Lond., A305:175-204, 1968.

[134] E. T. Newman and K. P. Tod. ASYMPTOTICALLY FLAT SPACE-TIMES. 1981.

[135] Ezra T. Newman and Theodore W. J. Unti. Behavior of Asymptotically Flat Empty Spaces. J. Math. Phys., 3(5):891, 1962.

[136] David A. Nichols. Center-of-mass angular momentum and memory effect in asymptotically flat spacetimes. Phys. Rev., D98(6):064032, 2018.

[137] Blagoje Oblak. BMS Particles in Three Dimensions. PhD thesis, Brussels U., 2016.

[138] Blagoje Oblak. Berry Phases on Virasoro Orbits. JHEP, 10:114, 2017. 
[139] Ioannis Papadimitriou. Holographic renormalization as a canonical transformation. JHEP, 11:014, 2010.

[140] Sabrina Pasterski. Asymptotic Symmetries and Electromagnetic Memory. JHEP, 09:154, 2017.

[141] Sabrina Pasterski, Shu-Heng Shao, and Andrew Strominger. Flat Space Amplitudes and Conformal Symmetry of the Celestial Sphere. Phys. Rev., D96(6):065026, 2017.

[142] Sabrina Pasterski, Andrew Strominger, and Alexander Zhiboedov. New Gravitational Memories. JHEP, 12:053, 2016.

[143] Monica Pate, Ana-Maria Raclariu, and Andrew Strominger. Color Memory: A Yang-Mills Analog of Gravitational Wave Memory. Phys. Rev. Lett., 119(26):261602, 2017.

[144] R. Penrose. Conformal treatment of infinity. Gen. Rel. Grav., 43:901-922, 2011. [,565(1964)].

[145] R. Penrose and W. Rindler. SPINORS AND SPACE-TIME. VOL. 2: SPINOR AND TWISTOR METHODS IN SPACE-TIME GEOMETRY. Cambridge Monographs on Mathematical Physics. Cambridge University Press, 1988.

[146] Roger Penrose. Asymptotic properties of fields and space-times. Phys. Rev. Lett., 10:66-68, 1963.

[147] Jiri Podolsky and Roland Steinbauer. Geodesics in space-times with expanding impulsive gravitational waves. Phys. Rev., D67:064013, 2003.

[148] Jiri Podolsky and Robert Svarc. Refraction of geodesics by impulsive spherical gravitational waves in constant-curvature spacetimes with a cosmological constant. Phys. Rev., D81:124035, 2010.

[149] Aaron Poole, Kostas Skenderis, and Marika Taylor. (A) $\mathrm{dS}_{4}$ in Bondi gauge. Class. Quant. Grav., 36(9):095005, 2019.

[150] Andrea Puhm. Conformally Soft Theorem in Gravity. 2019.

[151] Tullio Regge and Claudio Teitelboim. Role of Surface Integrals in the Hamiltonian Formulation of General Relativity. Annals Phys., 88:286, 1974.

[152] Max Riegler. How General Is Holography? PhD thesis, Vienna, Tech. U., 2016.

[153] Max Riegler and Céline Zwikel. Canonical Charges in Flatland. PoS, Modave2017:004, 2018.

[154] R. Sachs. Asymptotic symmetries in gravitational theory. Phys. Rev., 128:2851-2864, 1962.

[155] R. K. Sachs. Gravitational waves in general relativity. 8. Waves in asymptotically flat space-times. Proc. Roy. Soc. Lond., A270:103-126, 1962.

[156] Friedrich Scholler. Distinct Minkowski spaces from Bondi-Metzner-Sachs supertranslations. Phys. Rev., D97(4):046009, 2018.

[157] Kostas Skenderis. Lecture notes on holographic renormalization. Class. Quant. Grav., 19:5849-5876, 2002.

[158] Alexei A. Starobinsky. Isotropization of arbitrary cosmological expansion given an effective cosmological constant. JETP Lett., 37:66-69, 1983.

[159] Andrew Strominger. Black hole entropy from near horizon microstates. JHEP, 02:009, 1998.

[160] Andrew Strominger. Asymptotic Symmetries of Yang-Mills Theory. JHEP, 07:151, 2014.

[161] Andrew Strominger. On BMS Invariance of Gravitational Scattering. JHEP, 07:152, 2014.

[162] Andrew Strominger. Lectures on the Infrared Structure of Gravity and Gauge Theory. 2017. 
[163] Andrew Strominger and Alexander Zhiboedov. Gravitational Memory, BMS Supertranslations and Soft Theorems. JHEP, 01:086, 2016.

[164] Andrew Strominger and Alexander Zhiboedov. Superrotations and Black Hole Pair Creation. Class. Quant. Grav., 34(6):064002, 2017.

[165] Leonard Susskind. The World as a hologram. J. Math. Phys., 36:6377-6396, 1995.

[166] Leonard Susskind. Electromagnetic Memory. 2015.

[167] Gerard 't Hooft. Dimensional reduction in quantum gravity. Conf. Proc., C930308:284-296, 1993.

[168] Kip S. Thorne. Gravitational-wave bursts with memory: The Christodoulou effect. Phys. Rev., D45(2):520-524, 1992.

[169] Alexander Tolish, Lydia Bieri, David Garfinkle, and Robert M. Wald. Examination of a simple example of gravitational wave memory. Phys. Rev., D90(4):044060, 2014.

[170] Cedric Troessaert. Hamiltonian surface charges using external sources. J. Math. Phys., 57(5):053507, 2016.

[171] Robert M. Wald. Black hole entropy is the Noether charge. Phys. Rev., D48(8):R3427-R3431, 1993.

[172] Robert M. Wald and Andreas Zoupas. A General definition of 'conserved quantities' in general relativity and other theories of gravity. Phys. Rev., D61:084027, 2000.

[173] Steven Weinberg. Infrared photons and gravitons. Phys. Rev., 140:B516-B524, 1965.

[174] Steven Weinberg. The Quantum theory of fields. Vol. 1: Foundations. Cambridge University Press, 2005 .

[175] Chris D. White. Factorization Properties of Soft Graviton Amplitudes. JHEP, 05:060, 2011.

[176] J. Winicour. Global aspects of radiation memory. Class. Quant. Grav., 31:205003, 2014.

[177] Edward Witten. Anti-de Sitter space and holography. Adv. Theor. Math. Phys., 2:253-291, 1998.

[178] D. R. Yennie, Steven C. Frautschi, and H. Suura. The infrared divergence phenomena and high-energy processes. Annals Phys., 13:379-452, 1961.

[179] Ya. B. Zel'dovich and A. G. Polnarev. Radiation of gravitational waves by a cluster of superdense stars. sovast, 18:17, Aug 1974.

[180] Michael Zlotnikov. Sub-sub-leading soft-graviton theorem in arbitrary dimension. JHEP, 10:148, 2014. 